\title{
Inverse scattering transform for the defocusing nonlinear Schrödinger equation with fully asymmetric non-zero boundary conditions
}

\author{
Gino Biondini ${ }^{\mathrm{a}, \mathrm{b}}$, Emily Fagerstrom ${ }^{\mathrm{a}}$, Barbara Prinari ${ }^{\mathrm{c}, \mathrm{d}}$ \\ ${ }^{a}$ State University of New York at Buffalo, Department of Mathematics, Buffalo, NY 14260-2900 \\ ${ }^{b}$ State University of New York at Buffalo, Department of Physics, Buffalo, NY 14260-2900 \\ ${ }^{c}$ University of Colorado Colorado Springs, Department of Mathematics, Colorado Springs, CO USA 80918 \\ ${ }^{d}$ Università del Salento, Dipartimento di Matematica e Fisica "Ennio De Giorgi” and Sezione INFN, Lecce 73100, Italy
}

\begin{abstract}
We formulate the inverse scattering transform (IST) for the defocusing nonlinear Schrödinger (NLS) equation with fully asymmetric non-zero boundary conditions (i.e., when the limiting values of the solution at space infinities have different non-zero modulii). The theory is formulated without making use of Riemann surfaces, and instead by dealing explicitly with the branched nature of the eigenvalues of the associated scattering problem. For the direct problem, we give explicit single-valued definitions of the Jost eigenfunctions and scattering coefficients over the whole complex plane, and we characterize their discontinuous behavior across the branch cut arising from the square root behavior of the corresponding eigenvalues. We pose the inverse problem as a Riemann Hilbert Problem on an open contour, and we reduce the problem to a standard set of linear integral equations. Finally, for comparison purposes, we present the single-sheet, branch cut formulation of the inverse scattering transform for the initial value problem with symmetric (equimodular) non-zero boundary conditions, as well as for the initial value problem with one-sided non-zero boundary conditions, and we also briefly describe the formulation of the inverse scattering transform when a different choice is made for the location of the branch cuts.
\end{abstract}

Keywords: Inverse scattering transform, nonlinear Schrödinger equation, integrable systems, non-zero boundary conditions

\section{Introduction}

Integrable nonlinear evolution equations are physically relevant PDEs with a number of mathematically interesting properties (e.g., see $[1,2]$ and references therein). For example, they are completely integrable infinite-dimensional Hamiltonian systems; they have an infinite number of conserved quantities; they may be written as the compatibility condition of a Lax pair; the associated initial value problem (IVP) is solvable by the inverse scattering transform (IST) $[3,4]$. One of the most notable examples is the nonlinear Schrödinger (NLS) equation: it is a universal model for the evolution of the complex envelope of weakly nonlinear dispersive wave trains, and it appears in many different physical contexts, such as deep water waves, optics, acoustics, Bose-Einstein condensation, etc.

The aim of this work is to develop a consistent IST formalism for the defocusing NLS equation

$$
i q_{t}+q_{x x}-2|q|^{2} q=0
$$

Email addresses: biondini@buffalo.edu (Gino Biondini), emilyrf@buffalo.edu (Emily Fagerstrom), bprinari@uccs.edu (Barbara Prinari) 
(where subscripts $x, t$ denote partial differentiation) with fully asymmetric non-zero boundary conditions (NZBC):

$$
\lim _{x \rightarrow \pm \infty} q(x, t)=q_{ \pm}(t)
$$

with $\left|q_{+}(t)\right| \neq\left|q_{-}(t)\right|$, and with no condition on $\arg q_{ \pm}(t)$. Historically, most treatments of the NLS equation study the cases of zero boundary conditions (ZBC, i.e., with $q(x, t) \rightarrow 0$ as $x \rightarrow \pm \infty$ ) or symmetric NZBC (i.e., $q(x, t) \rightarrow q_{ \pm}(t)$, with $\left.q_{0}:=\left|q_{+}\right|=\left|q_{-}\right| \neq 0\right)$. In particular, see [2, 5, 6, 7, 8, 9] and references therein for the defocusing NLS with symmetric NZBC and $[10,11]$ for the focusing NLS with symmetric NZBC. However, in some physical applications one can also consider situations where the background amplitude is not the same in the two limits. For example, in nonlinear optics, asymmetric boundary conditions describe an input in which a continuous wave laser transitions gradually from one power value to another. Unfortunately, such situations are outside the scope of the traditional theory. A notable exception is a work by Boiti and Pempinelli [12], where the IST for the defocusing NLS with asymmetric NZBC was first proposed. However, the problem was formulated on a four-sheeted Riemann surface, which unnecessarily complicates the study. Moreover, no Riemann-Hilbert problem (RHP) was formulated and no characterization of the spectral data or of the solutions was given.

When the spatial derivatives of $q(x, t)$ vanish as $x \rightarrow \pm \infty,(1.1)$ implies $\left|q_{ \pm}(t)\right|=\left|q_{ \pm}(0)\right|$. Hence, without loss of generality we write the boundary conditions (BC) as

$$
q_{ \pm}(t)=A_{ \pm} \mathrm{e}^{i \alpha_{ \pm}-2 i A_{ \pm}^{2} t}
$$

with $\alpha_{ \pm} \in \mathbb{R}$ and $A_{ \pm}>0$. Moreover, thanks to the symmetry $x \mapsto-x$ of the NLS equation, we may take $A_{-}>A_{+}>0$ without loss of generality. For comparison purposes, we note that the BC considered in [12] included asymptotic plane-wave "carriers" $\mathrm{e}^{i \xi_{ \pm} x}$ as $x \rightarrow \pm \infty$. Importantly, however, in [12] the asymptotic carriers $\xi_{ \pm}$were the same (i.e., $\xi_{+}=\xi_{-}=\xi$ ), and in this case they could trivially be removed using the Galilean invariance of the NLS equation. Hence there is no loss of generality in setting $\xi=0$ and considering (1.3). Moreover, the BC considered in [12] did not include a phase shift, i.e., they were limited to the case $\alpha_{ \pm}=0$. Consequently, the BC considered in [12] are effectively a subset of (1.3).

The main result of this work is a constructive formalism to obtain the solution of the IVP for the defocusing NLS equation with the given NZBC (1.3) in terms of the scattering eigenfunctions. In turn, these eigenfunctions are obtained from the solution of an appropriate Riemann-Hilbert problem defined in terms of the scattering data. More precisely, the representation of the solution is summarized in the following theorem:

Theorem 1. Let $q(x, t)$ be the solution of the defocusing NLS equation (1.1) with NZBC (1.3), and assume that $(1+|\cdot|)\left(q(\cdot, t)-q_{ \pm}(t)\right) \in L^{1}\left(\mathbb{R}^{ \pm}\right)$and $\partial_{x} q(\cdot, t) \in L^{1}(\mathbb{R})$ for all $t \geqslant 0$. Given the solutions (2.8) of the first half of the Lax pair (2.1), let $\rho(k, t)$ be the corresponding reflection coefficient, determined for all $t \geqslant 0$ in terms the initial datum $q(x, 0)$ by (2.15) and (3.8); and let $k_{j}$, for $j=1, \ldots$, J be the discrete eigenvalues, determined by (2.40), with associated norming constants $C_{j}(t)$, determined for all $t \geqslant 0$ in terms of the initial datum $q(x, 0)$ by $(2.41)$ and (3.10). Then

$$
q(x, t)=-2 i \lim _{k \rightarrow \infty} k\left(N(x, t, k) E_{+}(k, t)\right)_{1,2},
$$

where double subscripts denote the corresponding matrix entries, $E_{+}(k, t)$ is defined in (2.7), and $N(x, t, k)$ is the solution of the following matrix Riemann-Hilbert problem:

(i) $N(x, t, k)$ is analytic for $k \in \mathbb{C} \backslash\left(\Sigma_{+} \cup K\right)$, where $\Sigma_{+}=\left(-\infty,-A_{+}\right] \cup\left[A_{+}, \infty\right)$ and $K=\left\{k_{1}, \ldots, k_{J}\right\} \subset$ $\left(-A_{+}, A_{+}\right)$

(ii) $N(x, t, k)=I+O(1 / k)$ as $k \rightarrow \infty$;

(iii) $N(x, t, k)$ satisfies the jump condition

$$
N^{+}(x, t, k)=N^{-}(x, t, k) \tilde{V}(x, t, k), \quad k \in \Sigma_{+},
$$

where the jump matrix $\tilde{V}(x, t, k)$ is defined in terms of $\rho(k, t)$ in (4.22), and is continuous $\forall k \in \Sigma_{+}$. 
(iv) For all $j=1, \ldots, J, N(x, t, k)$ has a simple pole at $k=k_{j}$ and satisfies the following residue conditions at $k=k_{j}$ :

$$
\begin{gathered}
\operatorname{Res}_{k=k_{j}}\left[N_{2}(x, t, k)-\frac{i q_{+}(t)}{k+\lambda_{+}(k)} N_{1}(x, t, k)\right]=0, \\
\operatorname{Res}_{k=k_{j}}\left[N_{1}(x, t, k)+\frac{i q_{+}^{*}(t)}{k+\lambda_{+}(k)} N_{2}(x, t, k)\right]=C_{j}(t) \mathrm{e}^{i\left(\lambda_{-, j}-\lambda_{+, j}\right) x}\left(N_{2}\left(x, t, k_{j}\right)-\frac{i q_{+}}{k_{j}+\lambda_{+, j}} N_{1}\left(x, t, k_{j}\right)\right),
\end{gathered}
$$

where single subscripts 1 and 2 denote the matrix columns, $\lambda_{ \pm, j}=\lambda_{ \pm}\left(k_{j}\right)$ and $\lambda_{ \pm}(k)$ are the unique, singlevalued functions defined for all $k \in \mathbb{C} \backslash \Sigma_{ \pm}$by (2.5) with $\operatorname{Im} \lambda_{ \pm}(k)>0$;

(v) $N(x, t, k)$ exhibits a square root singularity at $k= \pm A_{+}$, namely, $N(x, t, k)=O\left(k \mp A_{+}\right)^{1 / 2}$ as $k \rightarrow \pm A_{+}$, and is non-singular at $k= \pm A_{-}$.

The IST for the defocusing NLS equations with step initial data was used in $[13,14]$ to study the semiclassical limit and the short-time asymptotics of the solutions, respectively. (Similar problems for the focusing NLS equation were studied in [15, 16], and the long-time asymptotics of an initial-boundary value problem was considered in [17].) Note however that the case studied in [14] was with ZBC, and that the step IC considered in [13] are a special case of the general IC studied here.

The outline of this work is the following. In Section 2 we present the direct problem. (In particular, in Sections 2.2, 2.3 and 2.4 we discuss respectively the symmetries, the behavior at the branch points and the discrete spectrum.) In Section 3 we treat the time evolution. In Section 4 we formulate the inverse problem as a RHP and derive a representation for its solution in terms of scattering data. For comparison purposes, in Appendix A we present a formulation of the IST for the symmetric case with neither a uniformization variable nor a two-sheeted Riemann surface. (In particular, in A.3 we show how the reconstruction formula for the potential reduces to the one obtained with the uniformization variable). In Appendix B we present the solution of the same problem with a different choice of branch cut for the eigenvalue parameter. In Appendix C, as a further application of the methods presented in this work, we present the IST for the defocusing NLS equation with one-sided NZBC. In Appendix D we report the computation of the asymptotic behavior of the eigenfunctions as the scattering parameter tends to infinity, and in Appendix $\mathrm{E}$ we prove that the discrete eigenvalues are also simple in the case of asymmetric NZBC.

\section{Direct problem}

Recall that equation (1.1) is the compatibility condition of the Lax pair

$$
\Phi_{x}=X(x, t, k) \Phi, \quad \Phi_{t}=T(x, t, k) \Phi
$$

(the first of which is commonly referred to as the "scattering problem"), where

$$
X(x, t, k)=-i k \sigma_{3}+Q(x, t), \quad T(x, t, k)=-2 i k^{2} \sigma_{3}+i \sigma_{3}\left(Q_{x}-Q^{2}\right)+2 k Q,
$$

the potential and the Pauli matrices (introduced for later use) are

$$
Q(x, t)=\left(\begin{array}{cc}
0 & q(x, t) \\
q^{*}(x, t) & 0
\end{array}\right), \quad \sigma_{1}=\left(\begin{array}{ll}
0 & 1 \\
1 & 0
\end{array}\right), \quad \sigma_{2}=\left(\begin{array}{cc}
0 & -i \\
i & 0
\end{array}\right), \quad \sigma_{3}=\left(\begin{array}{cc}
1 & 0 \\
0 & -1
\end{array}\right),
$$

and the asterisk denotes complex conjugation. As $x \rightarrow \pm \infty$, one expects the solutions of the first of (2.1) to tend asymptotically to those of

$$
\Phi_{x}=X_{ \pm}(k, t) \Phi
$$

where

$$
X_{ \pm}(k, t)=-i k \sigma_{3}+Q_{ \pm}(t), \quad Q_{ \pm}(t)=\left(\begin{array}{cc}
0 & q_{ \pm}(t) \\
q_{ \pm}^{*}(t) & 0
\end{array}\right)
$$


The eigenvalues of $X_{ \pm}(k, t)$ are $\pm i \lambda_{ \pm}(k)$, where

$$
\lambda_{ \pm}^{2}=k^{2}-A_{ \pm}^{2}
$$

As expected, the eigenvalues have branching. In the symmetric case, $\lambda:=\lambda_{+}=\lambda_{-}$, and one can deal with this problem as in [4] by introducing a two-sheeted genus-0 Riemann surface which can be mapped back onto the complex plane via the uniformization variable $z=k+\lambda$. In the asymmetric case [i.e., $A_{+} \neq A_{-}$] however, $\lambda_{+}(k) \neq \lambda_{-}(k)$. The authors of [12] introduced a four-sheeted Riemann surface. Here we avoid the introduction of a Riemann surface altogether and define $\lambda_{ \pm}$as single-valued functions over a single copy of the complex plane. Of course these functions are discontinuous across their respective branch cuts, which affects the whole development of the IST.

\subsection{Eigenvalue branching, Jost solutions, analyticity and scattering matrix}

It will be useful in what follows to introduce the notations

$$
\Sigma_{ \pm}=\left(-\infty,-A_{ \pm}\right] \cup\left[A_{ \pm}, \infty\right), \quad \Sigma_{o}=\left[-A_{-},-A_{+}\right] \cup\left[A_{+}, A_{-}\right] .
$$

We will also make use of the corresponding interiors

$$
\stackrel{\circ}{\Sigma}_{ \pm}=\left(-\infty,-A_{ \pm}\right) \cup\left(A_{ \pm}, \infty\right), \quad \stackrel{\circ}{\Sigma}_{o}=\left(-A_{-}, A_{+}\right) \cup\left(A_{+}, A_{-}\right) .
$$

We uniquely define the eigenvalues $i \lambda_{ \pm}(k)$ as single-valued functions of $k \in \mathbb{C}$ using the same definition as [18]. Namely, we take the branch cuts for $\lambda_{ \pm}(k)$ on $\Sigma_{ \pm}$; for all $k \in \Sigma_{ \pm}$we take $\lambda_{ \pm}(k)$ to be the positive value of the real square root $\sqrt{k^{2}-A_{ \pm}^{2}}$, and for all $k \in \mathbb{C} \backslash \Sigma_{ \pm}$we define $\lambda_{ \pm}(k)$ as the single-valued, analytic functions which are continuous as $k$ approaches $\Sigma_{ \pm}$from above (i.e., as $\operatorname{Im} k \rightarrow 0^{+}$). Moreover, we emphasize that all eigenfunctions and scattering coefficients (to be defined below) that admit analytic extension from $\Sigma_{ \pm}$to the complex $k$-plane will have continuous projection to $\Sigma_{ \pm}$from above. Note that $\Sigma_{-} \subset \Sigma_{+}, \operatorname{Im} \lambda_{ \pm}(k) \geqslant 0$ for all $k \in \mathbb{C}$, and that both $\lambda_{ \pm}$are real-valued on $\Sigma_{-}$.

Similarly to $[9,6]$, a convenient choice for the eigenvector matrices of $X_{ \pm}(k, t)$ is

$$
E_{ \pm}(k, t)=I-\frac{i}{k+\lambda_{ \pm}(k)} \sigma_{3} Q_{ \pm}(t)
$$

We may then write two fundamental matrix solutions of the scattering problem as

$$
\Phi_{ \pm}(x, t, k)=E_{ \pm}(k, t) \mathrm{e}^{-i \lambda_{ \pm}(k) x \sigma_{3}}(I+o(1)), \quad k \in \stackrel{\circ}{\Sigma}_{ \pm}, \quad x \rightarrow \pm \infty .
$$

To emphasize, these Jost eigenfunctions are solutions of the scattering problem only; they are not solutions to both parts of the Lax pair. It will be useful to define

$$
d_{ \pm}(k)=\frac{1}{\operatorname{det} E_{ \pm}(k, t)}=\frac{k+\lambda_{ \pm}(k)}{2 \lambda_{ \pm}(k)}
$$

[Please see figure at the end of the document] 
and to note that

$$
E_{ \pm}^{-1}(k, t)=d_{ \pm}(k)\left[I+i \sigma_{3} Q_{ \pm}(t) /\left(k+\lambda_{ \pm}(k)\right)\right] .
$$

It can be shown that the Jost eigenfunctions are well-defined for $k \in \Sigma_{-}$, including at $\pm A_{-}$. These values comprise the continuous spectrum of the scattering problem; they are the values of $k$ for which both $\lambda_{ \pm}(k)$ are real-valued.

As in the case with zero BC at infinity, we remove the oscillations by introducing modified eigenfunctions

$$
\mu_{ \pm}(x, t, k)=\Phi_{ \pm}(x, t, k) \mathrm{e}^{i \lambda_{ \pm}(k) x \sigma_{3}} .
$$

The (modified) eigenfunctions can be rigorously defined as the unique solutions of the following integral equations:

$$
\begin{aligned}
& \mu_{-}(x, t, k)=E_{-}(k, t)+\int_{-\infty}^{x} E_{-}(k, t) \mathrm{e}^{-i \lambda_{-}(k)(x-y) \sigma_{3}} E_{-}^{-1}(k, t) \Delta Q_{-}(y, t) \mu_{-}(y, t, k) \mathrm{e}^{i \lambda_{-}(k)(x-y) \sigma_{3}} \mathrm{~d} y, \\
& \mu_{+}(x, t, k)=E_{+}(k, t)-\int_{x}^{\infty} E_{+}(k, t) \mathrm{e}^{-i \lambda_{+}(k)(x-y) \sigma_{3}} E_{+}^{-1}(k, t) \Delta Q_{+}(y, t) \mu_{+}(y, t, k) \mathrm{e}^{i \lambda_{+}(k)(x-y) \sigma_{3}} \mathrm{~d} y,
\end{aligned}
$$

where $\Delta Q_{ \pm}:=Q(x, t)-Q_{ \pm}(t)$, and analogously $\Delta q_{ \pm}:=q(x, t)-q_{ \pm}(t)$.

It is convenient to consider these integral equations columnwise, defining $\mu_{ \pm, 1}(x, t, k)$ and $\mu_{ \pm, 2}(x, t, k)$ to be the first and second column of $\mu_{ \pm}(x, t, k)$, respectively. Using a Neumann series for the integral equations for these columns, one can show that if $(1+|x|)\left(q(x, t)-q_{-}(t)\right) \in L^{1}\left(\mathbb{R}^{-}\right)$with respect to $x$ for all $t \geqslant 0$, then $\mu_{-, 1}(x, t, k)$ is well-defined on $\Sigma_{-}$and analytic in $\mathbb{C} \backslash \Sigma_{-}$. Similarly, if $(1+|x|)\left(q(x, t)-q_{+}(t)\right) \in L^{1}\left(\mathbb{R}^{+}\right)$with respect to $x$ for all $t \geqslant 0$, then $\mu_{+, 2}(x, t, k)$ is well-defined on $\Sigma_{+}$and analytic in $\mathbb{C} \backslash \Sigma_{+}$. Since the proof of these results is similar to the one in the symmetric case [7,9], it is omitted here for brevity. We should mention, however, that the condition $\left(q(x, t)-q_{ \pm}(t)\right) \in L^{1}\left(\mathbb{R}^{ \pm}\right)$is sufficient to guarantee analyticity of $\mu_{-, 1}(x, t, k)$ and $\mu_{+, 2}(x, t, k)$ in $\mathbb{C} \backslash \Sigma_{ \pm}$, respectively, and that the extra decay of the potential as $x \rightarrow \pm \infty$ is only required in order for the eigenfunctions to be well-defined at the branch points. Specifically, note that at the branch points $k= \pm A_{ \pm}$, one has $\lambda_{ \pm}(k)=0$, and $\operatorname{det} E_{ \pm}(k, t)=0$, which means that $E_{ \pm}(k, t)$ have no inverse at $k= \pm A_{ \pm}$. However, if $(1+|x|)\left(q(x, t)-q_{ \pm}(t)\right) \in L^{1}\left(\mathbb{R}^{ \pm}\right)$, the integral equations have well-defined limits as $\lambda_{ \pm}(k) \rightarrow 0$. To see this, observe that, for $\lambda_{-}(k) \neq 0$,

$$
E_{-}(k, t) \mathrm{e}^{-i \lambda_{-}(k)(x-y) \sigma_{3}} E_{-}^{-1}(k, t)=\frac{1}{\lambda_{-}(k)} \sin \left(\lambda_{-}(k)(x-y)\right) X_{-}(k, t)+\cos \left(\lambda_{-}(k)(x-y)\right) I .
$$

As $k \rightarrow \pm A_{-}$, the limit of the right hand side is $(x-y) X_{-}\left( \pm A_{-}, t\right)+I$, implying

$$
\mu_{-}\left(x, t, \pm A_{-}\right)=I \mp \sigma_{3} Q_{-}(t) / A_{-}+\int_{-\infty}^{x}\left[(x-y) X_{-}\left( \pm A_{-}, t\right)+I\right] \Delta Q_{-}(y, t) \mu_{-}\left(y, t, \pm A_{-}\right) \mathrm{d} y
$$

This is why the further condition that $x \Delta q_{-}(x, t) \in L^{1}\left(\mathbb{R}^{-}\right)$is needed in order for the above integral equation to admit a solution. An analogous argument holds for $\mu_{+}(x, t, k)$ at $k= \pm A_{+}$. The integral equations (2.11) will also be used to compute the asymptotics of the eigenfunctions for large $k$ in Appendix D.

Since $\operatorname{tr} X(x, t, k)=0$, Abel's formula implies that $\operatorname{det} \Phi_{ \pm}(x, t, k)$ is independent of $x$. Evaluating it in the limit $x \rightarrow \pm \infty$, we obtain

$$
\operatorname{det} \Phi_{ \pm}(x, t, k)=\operatorname{det} \mu_{ \pm}(x, t, k)=1 / d_{ \pm}(k) .
$$

For $k \in \Sigma_{\Sigma}^{\circ}$, both $\Phi_{ \pm}(x, t, k)$ are fundamental matrix solutions of the scattering ODE, and there exists a matrix $S(k, t)$, independent of $x$, such that

$$
\Phi_{-}(x, t, k)=\Phi_{+}(x, t, k) S(k, t), \quad k \in \Sigma_{-},
$$

with

$$
\operatorname{det} S(k, t)=d_{+}(k) / d_{-}(k) .
$$


Importantly, note that $\operatorname{det} S(k, t) \neq 1$, unlike the case of ZBC and symmetric NZBC. Note also that the scattering relation (2.12) also holds at $k= \pm A_{-}$, because $\Phi_{-}\left(x, t, \pm A_{-}\right)$is well-defined and $\Phi_{+}\left(x, t, \pm A_{-}\right)$is still a fundamental matrix solution of the scattering problem. On the other hand, $\operatorname{det} S\left( \pm A_{-}, t\right)=0$. (See Section 2.3 for further details.) The scattering coefficients, i.e., the entries $s_{i j}(k, t)$ of the scattering matrix $S(k, t)$, may be expressed as Wronskians:

$$
\begin{array}{ll}
s_{11}(k, t)=d_{+}(k) \operatorname{Wr}\left(\Phi_{-, 1}, \Phi_{+, 2}\right)(x, t, k), & s_{12}(k, t)=d_{+}(k) \operatorname{Wr}\left(\Phi_{-, 2}, \Phi_{+, 2}\right)(x, t, k), \\
s_{21}(k, t)=d_{+}(k) \operatorname{Wr}\left(\Phi_{+, 1}, \Phi_{-, 1}\right)(x, t, k), & s_{22}(k, t)=d_{+}(k) \operatorname{Wr}\left(\Phi_{+, 1}, \Phi_{-, 2}\right)(x, t, k) .
\end{array}
$$

The reflection coefficients we will use in the inverse problem are

$$
\rho(k, t):=s_{21}(k, t) / s_{11}(k, t), \quad \bar{\rho}(k, t):=s_{12}(k, t) / s_{22}(k, t), \quad k \in \Sigma_{-} .
$$

Using the Wronskian definitions (2.14), we may analytically continue $s_{11}(k, t)$ off the continuous spectrum. Specifically, $s_{11}(k, t)$ is analytic for $k \in \mathbb{C} \backslash \Sigma_{+}$. Moreover, since $\Phi_{-, 1}(x, t, k)$ may be analytically extended to $\mathbb{C} \backslash \Sigma \Sigma_{-}$and $\Phi_{+, 1}(x, t, k)$ is defined on $\Sigma_{+}$, we may extend the definition of $s_{21}(k, t)$ pointwise to $\Sigma_{+}^{\circ}$. In fact, the first column of the scattering relation (2.12) continues to hold on $\Sigma_{+}^{\circ}$. However, in Section 2.3 we will show that generically $s_{11}(k, t)$ and $s_{21}(k, t)$ have singularities at $k= \pm A_{+}$[where $d_{+}(k)$ has a square root singularity]. On the other hand, we will also show that $\rho(k, t)$ has finite limits as $k \rightarrow \pm A_{+}$.

\subsection{Symmetries}

We now consider the symmetries of the eigenfunctions and scattering data. There are two kinds of symmetries, corresponding to the involutions $k \mapsto k^{*}$ and $\lambda_{ \pm}(k) \mapsto-\lambda_{ \pm}(k)$, respectively.

First symmetry. The first symmetry, corresponding to the involution $k \mapsto k^{*}$, is analogous to the one in the case of ZBC. If a $2 \times 2$ matrix $v(x, t, k)$ solves the scattering problem, so does $\hat{v}(x, t, k):=\sigma_{1} v^{*}\left(x, t, k^{*}\right) \sigma_{1}$, where the asterisk denotes complex conjugation and not the matrix adjoint. Considering the above relation for $k \in \mathbb{R}$ and comparing asymptotic behaviors as $x \rightarrow \pm \infty$, we have:

$$
\Phi_{ \pm}(x, t, k)=\sigma_{1} \Phi_{ \pm}^{*}(x, t, k) \sigma_{1}, \quad k \in \Sigma_{ \pm}
$$

(To avoid any possible confusion, we emphasize that the above relation is evaluated pointwise for $k \in \Sigma_{ \pm} \subset \mathbb{R}$, where the corresponding eigenfunctions are defined. In other words, the matrix $\Phi_{ \pm}^{*}(x, t, k)$ appearing in the right hand side does not equal $\lim _{k \rightarrow \mathbb{R}} \Phi_{ \pm}^{*}\left(x, t, k^{*}\right)$. In fact, since only one of the columns of the matrix $\Phi_{ \pm}(x, t, k)$ can be analytically extended off the real axis, the Schwarz conjugate inside the above limit does not exist in general.) Substituting the previous equations into (2.12), we have $S(k, t)=\sigma_{1} S^{*}(k, t) \sigma_{1}$ for $k \in \Sigma_{-}$. That is,

$$
s_{11}(k, t)=s_{22}^{*}(k, t), \quad s_{21}(k, t)=s_{12}^{*}(k, t), \quad k \in \Sigma_{-},
$$

which in turn implies

$$
\bar{\rho}(k, t)=\rho^{*}(k, t), \quad k \in \Sigma_{-} .
$$

Note also that (2.13) implies

$$
\left|s_{11}(k, t)\right|^{2}-\left|s_{21}(k, t)\right|^{2}=d_{+}(k) / d_{-}(k), \quad k \in \Sigma_{-} .
$$

Since $d_{+}(k) / d_{-}(k)>0$ for $k \in \stackrel{\Sigma}{\Sigma}_{-}$, we conclude that $s_{11}(k, t)$ has no zeros on $\Sigma^{\circ}-\left(\right.$ recall $\Sigma_{ \pm}^{\circ}$ and $\Sigma_{0}^{\circ}$ were defined in (2.6)). We will also show in Section 2.3 that at $k= \pm A_{-}$one has $\left|s_{11}\left( \pm A_{-}, t\right)\right|=\left|s_{21}\left( \pm A_{-}, t\right)\right| \neq 0$. For future reference, we note that (2.19) can also be written in terms of the reflection coefficient as follows:

$$
\left|s_{11}(k, t)\right|^{2}=\left(1-|\rho(k, t)|^{2}\right)^{-1} d_{-}(k) / d_{+}(k), \quad k \in \Sigma_{-} .
$$


We also consider $k \mapsto k^{*}$ for $k \notin \Sigma_{+}$. Again, if $v(x, t, k)$ is a vector solution to the scattering problem, then $\sigma_{1} v^{*}\left(x, t, k^{*}\right)$ is as well. Looking at the asymptotic behavior of $\sigma_{1} \Phi_{-, 1}^{*}\left(x, t, k^{*}\right)$ as $x \rightarrow-\infty$ for $k \notin \Sigma_{+}$, we have

$$
\sigma_{1} \Phi_{-, 1}^{*}\left(x, t, k^{*}\right)=\frac{-i q_{-}(t)}{k-\lambda_{-}(k)} \Phi_{-, 1}(x, t, k), \quad k \notin \Sigma_{+}
$$

Similarly, looking at the behavior as $x \rightarrow \infty$,

$$
\sigma_{1} \Phi_{+, 2}^{*}\left(x, t, k^{*}\right)=\frac{i q_{+}^{*}(t)}{k-\lambda_{+}(k)} \Phi_{+, 2}(x, t, k), \quad k \notin \Sigma_{+} .
$$

Finally, using the Wronskian expressions $(2.14)$ we may determine $s_{11}^{*}\left(k^{*}, t\right)$ when $k \notin \Sigma_{+}$:

$$
s_{11}^{*}\left(k^{*}, t\right)=\frac{q_{-}(t)}{q_{+}(t)} \frac{k-\lambda_{+}(k)}{k-\lambda_{-}(k)} s_{11}(k, t), \quad k \notin \Sigma_{+} .
$$

Second symmetry. The second symmetry corresponds to the involution $\lambda_{ \pm}(k) \mapsto-\lambda_{ \pm}(k)$, To derive this symmetry, note that in defining $\lambda_{ \pm}(k)$, we could have taken the opposite sign of the complex square roots. The integral equations for the Jost eigenfunctions are formally independent of this choice. However, the resulting eigenfunctions $d o$ depend on the choice of sign. With some abuse of notation, let us temporarily express the dependence of the Jost eigenfunctions on the choice of sign explicitly. From the above discussion we have that if $\Phi_{ \pm}\left(x, t, k, \lambda_{ \pm}(k)\right)$ solves the first of the Lax pair (2.2), then so does $\tilde{\Phi}_{ \pm}(x, t, k):=\Phi_{ \pm}\left(x, t, k,-\lambda_{ \pm}(k)\right)$.

Alternatively, one could introduce the eigenfunctions $\tilde{\Phi}_{ \pm}(x, t, k)$ without considering the change $\lambda_{ \pm}(k) \mapsto$ $-\lambda_{ \pm}(k)$ by defining them as the solution of the scattering problem such that

$$
\tilde{\Phi}_{ \pm}(x, t, k)=\left(I-\frac{i}{k-\lambda_{ \pm}(k)} \sigma_{3} Q_{ \pm}(t)\right) \mathrm{e}^{i \lambda_{ \pm}(k) x \sigma_{3}}(1+o(1)) \quad \text { as } x \rightarrow \pm \infty .
$$

Since $\Phi_{ \pm}(x, t, k)$ and $\tilde{\Phi}_{ \pm}(x, t, k)$ are fundamental matrix solutions of the scattering problem for all $k \in \Sigma^{\circ}$, one can express one set of solutions in terms of the other via a matrix independent of $x$. Comparing asymptotics as $x \rightarrow \pm \infty$, we have:

$$
\begin{aligned}
& \tilde{\Phi}_{ \pm}(x, t, k)=\Phi_{ \pm}(x, t, k) \frac{-i}{k-\lambda_{ \pm}(k)} \sigma_{3} Q_{ \pm}(t), \quad k \in \stackrel{\circ}{\Sigma}_{ \pm} \\
& \Phi_{ \pm}(x, t, k)=\tilde{\Phi}_{ \pm}(x, t, k) \frac{-i}{k+\lambda_{ \pm}(k)} \sigma_{3} Q_{ \pm}(t), \quad k \in \stackrel{\circ}{\Sigma}_{ \pm} .
\end{aligned}
$$

Denoting by $\tilde{S}(k, t)$ the scattering matrix for $\tilde{\Phi}_{ \pm}(x, t, k)$, we have

$$
S(k, t)=\frac{k-\lambda_{-}(k)}{k-\lambda_{+}(k)} \sigma_{3} Q_{+}(t) \tilde{S}(k, t) Q_{-}^{-1}(t) \sigma_{3}, \quad k \in \Sigma_{-} .
$$

Or, componentwise,

$$
s_{22}(k, t)=\frac{q_{+}^{*}(t)}{q_{-}^{*}(t)} \frac{k-\lambda_{-}(k)}{k-\lambda_{+}(k)} \tilde{s}_{11}(k, t), \quad s_{21}(k, t)=-\frac{q_{+}^{*}(t)}{q_{-}(t)} \frac{k-\lambda_{-}(k)}{k-\lambda_{+}(k)} \tilde{s}_{12}(k, t), \quad k \in \Sigma_{-} .
$$

As we show next, these relations will be useful when considering the limits of the analytic columns of the Jost solutions as $k$ approaches the branch cuts from below.

Limits of the eigenfunctions and scattering coefficients from below the branch cuts. Recall that $\lambda_{ \pm}(k)$ are analytic in $\mathbb{C} \backslash \Sigma_{ \pm}$and discontinuous across their respective branch cuts. In particular, $\lambda_{ \pm}(k)$ were defined to be continuous as $k \rightarrow \Sigma_{ \pm}$from above, namely

$$
\lambda_{ \pm}^{+}(k):=\lim _{\epsilon \downarrow 0} \lambda_{ \pm}(k+i \epsilon)=\lambda_{ \pm}(k)
$$


Conversely, we have the following limits as $k$ approaches $\Sigma_{ \pm}$from below:

whereas

$$
\lambda_{ \pm}^{-}(k):=\lim _{\epsilon \uparrow 0} \lambda_{ \pm}(k+i \epsilon)=-\lambda_{ \pm}(k), \quad k \in \Sigma_{-},
$$

$$
\begin{gathered}
\lambda_{-}^{-}(k):=\lim _{\epsilon \uparrow 0} \lambda_{-}(k+i \epsilon)=\lambda_{-}(k), \quad k \in \Sigma_{o}, \\
\lambda_{+}^{-}(k):=\lim _{\epsilon \uparrow 0} \lambda_{+}(k+i \epsilon)=-\lambda_{+}(k), \quad k \in \Sigma_{o} .
\end{gathered}
$$

Similarly, $\Phi_{-, 1}(x, t, k)$ is analytic for $k \in \mathbb{C} \backslash \Sigma_{-}$and is continuous to $\Sigma_{-}$from above, and $\Phi_{+, 2}(x, t, k)$ is analytic for $k \in \mathbb{C} \backslash \Sigma_{+}$and is continuous to $\Sigma_{+}$from above. Conversely, we have the following limits as $k \rightarrow \Sigma_{ \pm}$from below:

$$
\begin{array}{ll}
\Phi_{-, 1}^{-}(x, t, k):=\lim _{\epsilon \uparrow 0} \Phi_{-, 1}(x, t, k+i \epsilon)=\tilde{\Phi}_{-, 1}(x, t, k), & k \in \Sigma_{-}, \\
\Phi_{+, 2}^{-}(x, t, k):=\lim _{\epsilon \uparrow 0} \Phi_{+, 2}(x, t, k+i \epsilon)=\tilde{\Phi}_{+, 2}(x, t, k), & k \in \Sigma_{-}, \\
\Phi_{-, 1}^{-}(x, t, k):=\lim _{\epsilon \uparrow 0} \Phi_{-, 1}(x, t, k+i \epsilon)=\Phi_{-, 1}(x, t, k), & k \in \Sigma_{o} \\
\Phi_{+, 2}^{-}(x, t, k):=\lim _{\epsilon \uparrow 0} \Phi_{+, 2}(x, t, k+i \epsilon)=\tilde{\Phi}_{+, 2}(x, t, k), & k \in \Sigma_{o} .
\end{array}
$$

In other words, the above columns of $\tilde{\Phi}_{ \pm}(x, t, k)$ evaluated on the branch cuts coincide with the limit of the analytic columns of $\Phi_{ \pm}(x, t, k)$ as they approach the branch cuts from below. In particular, using the symmetry relations (2.24), we have

$$
\begin{aligned}
& \Phi_{-, 1}^{-}(x, t, k)=\frac{i q_{-}^{*}(t)}{k-\lambda_{-}} \Phi_{-, 2}(x, t, k), \quad k \in \Sigma_{-}, \\
& \Phi_{+, 2}^{-}(x, t, k)=\frac{-i q_{+}(t)}{k-\lambda_{+}} \Phi_{+, 1}(x, t, k), \quad k \in \Sigma_{+} .
\end{aligned}
$$

Since $\Phi_{-, 1}(x, t, k)$ and $\Phi_{+, 2}(x, t, k)$ may be analytically extended in $k$ to $\mathbb{C} \backslash \Sigma_{+}, s_{11}(k, t)$ may be extended as well using the Wronskian definitions (2.14). We can then use the above relations to compute the limits of $s_{11}(k, t)$ as $k$ approaches $\Sigma_{ \pm}$from below:

$$
\begin{aligned}
& s_{11}^{-}(k, t):=\lim _{\epsilon \uparrow 0} s_{11}(k+i \epsilon, t)=\frac{q_{+}(t)}{q_{-}(t)} \frac{k+\lambda_{-}(k)}{k+\lambda_{+}(k)} s_{22}(k, t), \quad k \in \Sigma_{-} \\
& s_{11}^{-}(k, t):=\lim _{\epsilon \uparrow 0} s_{11}(k+i \epsilon, t)=-\frac{i q_{+}(t)}{k+\lambda_{+}(k)} s_{21}(k, t), \quad k \in \stackrel{\circ}{\Sigma}_{o} .
\end{aligned}
$$

The limits to the branch cuts from below of $\mu_{-, 1}(x, t, k)$ and $\mu_{+, 2}(x, t, k)$, which will be used in the inverse problem, are obtained from (2.27), (2.28), and (2.29) using the definition (2.10).

\subsection{Behavior of the scattering coefficients at the branch points}

In the IVP with symmetric NZBC, each of the scattering coefficients has a square root singularity at each of the two branch points, and at the same time all the scattering coefficients become proportional (since the determinant of the scattering matrix vanishes in the limit). In the IVP with asymmetric NZBC, as we have seen, there are two sets of branch points, $\pm A_{-}$and $\pm A_{+}$, and these features are decoupled in general.

We have seen in Section 2.1 that, at $k= \pm A_{-}$, both $\Phi_{+}(x, t, k)$ and $\Phi_{-}(x, t, k)$ are well defined and the scattering relation (2.12) still holds, since $\Phi_{+}\left(x, t, \pm A_{-}\right)$is still a fundamental matrix solution. As a consequence, all entries of the scattering matrix $S(k, t)$ are well defined at $k= \pm A_{-}$. Since $1 / d_{-}(k) \rightarrow 0$ as $k \rightarrow \pm A_{-}$, however, the columns of $\Phi_{-}\left(x, t, \pm A_{-}\right)$are linearly dependent, so $\operatorname{det} S\left( \pm A_{-}, t\right)=0$, and the scattering coefficients at $k= \pm A_{-}$ 
are therefore not independent from each other. Specifically, comparing the asymptotics of $\Phi_{-, 1}\left(x, t, \pm A_{-}\right)$and $\Phi_{-, 2}\left(x, t, \pm A_{-}\right)$as $x \rightarrow-\infty$, we have

$$
s_{11}\left( \pm A_{-}, t\right)= \pm i \mathrm{e}^{-i \alpha_{-}+2 i A_{-}^{2} t} s_{12}\left( \pm A_{-}, t\right), \quad s_{21}\left( \pm A_{-}, t\right)= \pm i \mathrm{e}^{-i \alpha_{-}+2 i A_{-}^{2} t} s_{22}\left( \pm A_{-}, t\right),
$$

with $\alpha_{-}$defined in (1.3). Note also that from (2.17) and (2.31) it follows that

$$
\left|s_{11}\left( \pm A_{-}, t\right)\right|=\left|s_{21}\left( \pm A_{-}, t\right)\right| \neq 0 .
$$

[This is because, if one of the scattering coefficients were zero, they would all be zero due to their symmetries, implying $\Phi_{-}\left(x, t, \pm A_{-}\right) \equiv 0 \forall x \in \mathbb{R}$, in contradiction with (2.8)]. Thus, it follows that

$$
\left|\rho\left( \pm A_{-}, t\right)\right|=1 \text {. }
$$

Note that, unlike the case of symmetric NZBC, (2.33) is consistent with the fact that $s_{11}\left( \pm A_{-}, t\right)$ is finite, thanks to the factor $d_{-}(k)$ in (2.20).

The situation is different at $k= \pm A_{+}$, since the Jost eigenfunctions $\Phi_{+}(x, t, k)$ are continuous there, but only one of the columns of $\Phi_{-}(x, t, k)$ [namely, $\left.\Phi_{-, 1}(x, t, k)\right]$ is defined there. Also recall that only the scattering coefficients $s_{11}(k, t)$ and $s_{21}(k, t)$ are defined for $k \in \Sigma_{o}^{\prime}$. To compute their limits as $k \rightarrow \pm A_{+}$, note first that

$$
d_{+}(k)=\frac{\left( \pm A_{+}\right)^{1 / 2}}{2 \sqrt{2}\left(k \mp A_{+}\right)^{1 / 2}}+\frac{1}{2}+O\left(k \mp A_{+}\right)^{1 / 2}, \quad k \rightarrow \pm A_{+} .
$$

The Wronskian relations (2.14) then imply that, generically, $s_{11}(k, t)$ and $s_{21}(k, t)$ have square root singularities, since

$$
\begin{array}{ll}
s_{11}(k, t)=d_{+}(k)\left(\operatorname{Wr}\left(\Phi_{-, 1}, \Phi_{+, 2}\right)\left(x, t, \pm A_{+}\right)+o(1)\right), & k \rightarrow \pm A_{+}, \\
s_{21}(k, t)=d_{+}(k)\left(\operatorname{Wr}\left(\Phi_{+, 1}, \Phi_{-, 1}\right)\left(x, t, \pm A_{+}\right)+o(1)\right), & k \rightarrow \pm A_{+} .
\end{array}
$$

On the other hand, the reflection coefficient $\rho(k, t)$, which is still defined for all $k \in \stackrel{\circ}{\Sigma}_{o}$ via (2.15), remains finite as $k \rightarrow \pm A_{+}$. Indeed, comparing the asymptotics of $\Phi_{+, 1}\left(x, t, \pm A_{+}\right)$and $\Phi_{+, 2}\left(x, t, \pm A_{+}\right)$as $x \rightarrow \infty$, we have

$$
\lim _{k \rightarrow \pm A_{+}} \rho(k, t)=\mp i e^{-i \alpha_{+}+2 i A_{+}^{2} t},
$$

implying $\left|\rho\left( \pm A_{+}, t\right)\right|=1$, as with the behavior as $k \rightarrow \pm A_{-}$.

\subsection{Discrete spectrum}

We have seen that values of $k \in \Sigma_{-}$constitute the continuous spectrum of the scattering problem. Any values of $k \notin \Sigma_{-}$for which $L^{2}$ eigenfunctions exist will constitute the discrete spectrum of the scattering problem. Since the scattering operator is self-adjoint, any discrete eigenvalues must be real, and must therefore lie in the segment $\left(-A_{-}, A_{-}\right)$. In addition, in the development of the inverse problem one must also take into account any so-called "spectral singularities", namely, values of $k$ for which the jump matrix of the Riemann-Hilbert problem is singular. Such values correspond to zeros of one of the scattering coefficients. Next we discuss the possible locations of such zeros.

Specifically, recall that in the previous sections we have shown that $s_{11}(k, t) \neq 0 \forall k \in \Sigma_{-}$, which ensures that no spectral singularities can arise in this region (similarly to the case of symmetric NZBC, but now also including the points $k= \pm A_{-}$). For $k \notin \Sigma_{-}$, either or both of $\lambda_{ \pm}(k)$ are not real, and the solutions of the scattering problem are in general unbounded over the whole real $x$-axis. As in the case with ZBC, bounded solutions exist when the Jost eigenfunction that vanishes as $x \rightarrow-\infty$ [namely, $\Phi_{-, 1}(x, t, k)$ ] is proportional to an eigenfunction that vanishes or is bounded as $x \rightarrow \infty$; namely, $\Phi_{+, 1}(x, t, k)$ for $k \in \Sigma^{\circ}$ or $\Phi_{+, 2}(x, t, k)$ for $k \in\left[-A_{-}, A_{-}\right]$. The Wronskian relations (2.14) imply that this occurs when $s_{21}(k, t)=0$ or $s_{11}(k, t)=0$, respectively. 
Non-existence of discrete eigenvalues in $\Sigma_{0}^{\circ}$. For $k \in \stackrel{\Sigma}{\Sigma}_{0}$, one has $\lambda_{-}(k) \in i \mathbb{R}^{+}$but $\lambda_{+}(k) \in \mathbb{R}$. Thus, even if $\Phi_{-, 1}\left(x, t, k_{o}\right)$ and $\Phi_{+, 2}\left(x, t, k_{o}\right)$ were proportional for some $k_{o}$ in this region [corresponding to $s_{11}\left(k_{o}\right)=0$ ], one would not obtain a bound state, since $\Phi_{+, 2}\left(x, t, k_{0}\right)$ does not tend to zero as $x \rightarrow \infty$. Nonetheless, any such zeros would need to be taken into account in the inverse problem. Importantly, however, we next show that

$$
s_{11}(k, t) s_{21}(k, t) \neq 0 \quad \forall k \in \stackrel{\circ}{\Sigma}_{0} .
$$

To prove (2.37), note first that, for any two solutions $u(x, t, k)$ and $v(x, t, k)$ of the scattering problem,

$$
\frac{\partial}{\partial x}\left(u^{\dagger} \sigma_{3} v\right)=0
$$

where + denotes conjugate transpose. Recalling the pointwise symmetry (2.16) we have, $\forall k \in \Sigma_{+}, \Phi_{+, 1}(x, t, k)=$ $\sigma_{1} \Phi_{+, 2}^{*}(x, t, k)$ and $\Phi_{+, 2}(x, t, k)=\sigma_{1} \Phi_{+, 1}^{*}(x, t, k)$. Then, setting $u=v=\Phi_{+, 1}\left(x, t, k_{o}\right)$ or $u=v=\Phi_{+, 2}\left(x, t, k_{o}\right)$ for arbitrary $k_{0} \in \stackrel{\Sigma}{\Sigma}_{0}$, using the above symmetries and evaluating $u^{\dagger} \sigma_{3} v$ as $x \rightarrow \infty$ we obtain

$$
\Phi_{+, j}^{+}\left(x, t, k_{0}\right) \sigma_{3} \Phi_{+, j}\left(x, t, k_{0}\right)=1 / d_{+}\left(k_{o}\right) \quad \forall x \in \mathbb{R}, \quad k_{o} \in \stackrel{\circ}{\Sigma}_{o}, \quad j=1,2 .
$$

Now suppose that $s_{11}\left(k_{o}\right)=0$ or $s_{21}\left(k_{o}\right)=0$. The Wronskian relations (2.14) imply, respectively, $\Phi_{+, 2}\left(x, t, k_{0}\right)=$ $c_{0} \Phi_{-, 1}\left(x, t, k_{0}\right)$ or $\Phi_{+, 1}\left(x, t, k_{0}\right)=c_{0} \Phi_{-, 1}\left(x, t, k_{0}\right)$ for some non-zero constant $c_{0} \in \mathbb{C}$. On the other hand, $\Phi_{-, 1}(x, t, k) \rightarrow 0$ as $x \rightarrow-\infty \forall k \in\left(-A_{-}, A_{-}\right) \supset \stackrel{\circ}{\Sigma}_{0}$. Therefore, the left-hand side of (2.39) also tends to 0 as $x \rightarrow-\infty$, resulting in a contradiction, since $1 / d_{+}\left(k_{0}\right) \neq 0 \forall k_{0} \in \Sigma_{0}^{\circ}$. Thus $s_{11}(k, t)$ and $s_{21}(k, t)$ must both be non-zero for all $k \in \stackrel{\Sigma}{\Sigma}_{0}$.

Importantly, it follows from (2.37) that $\rho(k, t)$ and $1 / \rho(k, t)$ [as defined in (2.15)] have no zeros or poles in $\stackrel{\circ}{\Sigma}_{o}$, which will ensure that the jump matrix of the RHP is always non-singular. Also, the same result implies that it is impossible to have $\rho(k, t) \equiv 0$ for all $k \in \stackrel{\Sigma}{\Sigma}_{0}$, i.e., no pure reflectionless solutions are possible in the IVP with asymmetric NZBC. Of course one could still have situations in which $\rho(k, t) \equiv 0$ for all $k \in \Sigma_{-}$. Such cases do not lead to pure soliton solutions, however, because the inverse problem would still have a contribution arising from the jump across $k \in \Sigma_{\Sigma}^{\circ}$.

Discrete eigenvalues in $\left(-A_{+}, A_{+}\right)$. As we have shown above, $s_{11}(k, t) \neq 0 \forall k \in \stackrel{\Sigma}{\Sigma}_{+}$. Also, as shown in Section 2.3, generically it has a square root singularity at $k= \pm A_{+}$. Thus, any zeros of $s_{11}(k, t)$ lie in $\left(-A_{+}, A_{+}\right)$, for which $\lambda_{ \pm}(k)$ are both purely imaginary. Throughout the rest of this work, we will assume that there are a finite number of such zeros. A sufficient condition for this to happen is that $s_{11}\left( \pm A_{+}, t\right) \neq 0$. [Recall that $s_{11}(k, t)$ is analytic in a region containing $\left(-A_{+}, A_{+}\right)$. If $s_{11}(k, t)$ had an infinite number of zeros in that interval, there would be an accumulation point of zeros in the closure of the interval. Such an accumulation point cannot be in the interior of the interval, however, because otherwise $s_{11}(k, t)$ would be identically zero. Thus, the only possibility for $s_{11}(k, t)$ to have an infinite number of zeros is if either $s_{11}\left(A_{+}, t\right)$ or $s_{11}\left(-A_{+}, t\right)$ are zero. In the symmetric case, the situation when $s_{11}(k, t)=0$ at either branch point is called a virtual level [8].] Note, however, that the condition $s_{11}\left( \pm A_{+}, t\right) \neq 0$ is sufficient but not necessary to ensure a finite number of zeros. [E.g., all reflectionless solutions of the symmetric case correspond to virtual levels.]

Denoting by $k_{1}, \ldots, k_{j}$ the zeros of $s_{11}(k, t)$ in $\left(-A_{+}, A_{+}\right)$, we write $\lambda_{ \pm, j}:=\lambda_{ \pm}\left(k_{j}\right)$. At $k=k_{j}$, we have

$$
\Phi_{-, 1}\left(x, t, k_{j}\right)=b_{j}(t) \Phi_{+, 2}\left(x, t, k_{j}\right), \quad j=1, \ldots, J,
$$

for some constants $b_{j}$. In the inverse problem we make use of the norming constants

$$
C_{j}(t)=b_{j}(t) / s_{11}^{\prime}\left(k_{j}, t\right),
$$

where prime denotes differentiation with respect to $k$. Note that, as in the case of symmetric NZBC [8], one can show that any zeros of $s_{11}(k, t)$ are simple, i.e., $s_{11}\left(k_{j}, t\right)=0$ implies $s_{11}^{\prime}\left(k_{j}, t\right) \neq 0$ (see Appendix E for a proof).

Recalling (2.21) produces the relation

$$
b_{j}(t)=-\frac{q_{+}^{*}(t)}{q_{-}(t)} \frac{k_{j}-\lambda_{-, j}}{k_{j}-\lambda_{+, j}} b_{j}^{*}(t), \quad j=1, \ldots, J,
$$


and applying (2.22), we may write

$$
\left[s_{11}^{\prime}\left(k_{j}, t\right)\right]^{*}=\frac{q_{-}(t)}{q_{+}(t)} \frac{k_{j}-\lambda_{+, j}}{k_{j}-\lambda_{-, j}} s_{11}^{\prime}\left(k_{j}, t\right), \quad j=1, \ldots, J .
$$

Thus the norming constants satisfy the constraint

$$
C_{j}^{*}(t)=-C_{j}(t)\left[q_{+}(t) / q_{+}^{*}(t)\right], \quad j=1, \ldots, J .
$$

\section{Time evolution}

Recall that in the symmetric case (where $A_{+}=A_{-}=q_{0}$ ) the time dependence of the boundary conditions can be eliminated by performing the rescaling $q(x, t)=\tilde{q}(x, t) \exp \left(-2 i q_{0}^{2} t\right)$ which adds the term $+2 q_{0}^{2} q$ to (1.1). This has the advantage that one can easily define the Jost eigenfunctions to be simultaneous solutions of both parts of the Lax pair, which in turn makes the scattering data independent of time and therefore allows one to take into account the time evolution of the problem in a simpler way (e.g., see [6]).

In the asymmetric case, such a rescaling is not possible. In light of this, the asymptotic behavior for the Jost solutions was chosen to be fixed and independent of time [apart from a trivial parametric dependence via $E_{ \pm}(k, t)$ ]. As a result, $\Phi_{-}(x, t, k)$ and $\Phi_{+}(x, t, k)$ are not solutions of the second half of the Lax pair. Nevertheless the NLS equation is the compatibility condition of the Lax pair, so simultaneous solutions $\Psi(x, t, k)$ of both parts of the Lax pair must exist. Since $\Phi_{ \pm}(x, t, k)$ are both fundamental matrix solutions of the scattering problem for all $k \in \Sigma_{ \pm}^{\circ}$, we can express $\Psi(x, t, k)$ in terms of them via matrices $C_{ \pm}(k, t)$ independent of $x$. That is,

$$
\Psi(x, t, k)=\Phi_{ \pm}(x, t, k) C_{ \pm}(k, t), \quad k \in \Sigma_{ \pm}^{\circ} .
$$

Differentiating (3.1) with respect to time, we have

$$
\left(C_{ \pm}\right)_{t}=R_{ \pm} C_{ \pm}, \quad k \in \stackrel{\circ}{\Sigma}_{ \pm}
$$

with

$$
R_{ \pm}(k, t)=\Phi_{ \pm}^{-1}\left[T \Phi_{ \pm}-\left(\Phi_{ \pm}\right)_{t}\right], \quad k \in \stackrel{\circ}{\Sigma}_{ \pm} .
$$

Since $R_{ \pm}(k, t)$ and $C_{ \pm}(k, t)$ are independent of $x$, we may evaluate them in the limit as $x \rightarrow \pm \infty$ via (2.8):

$$
R_{ \pm}(k, t)=\lim _{x \rightarrow \pm \infty} \Phi_{ \pm}^{-1}\left(T \Phi_{ \pm}-\left(\Phi_{ \pm}\right)_{t}\right)=i f_{ \pm}(k) \sigma_{3}, \quad k \in \Sigma_{ \pm}^{\circ},
$$

where $f_{ \pm}(k)=A_{ \pm}^{2}+2 k \lambda_{ \pm}(k)$. Conversely, from (3.3) we can obtain the time evolution of the Jost solutions as

$$
\left(\Phi_{ \pm}\right)_{t}=T \Phi_{ \pm}-\Phi_{ \pm} R_{ \pm}, \quad k \in \Sigma_{ \pm} .
$$

[Note (3.5) can be extended to the corresponding branch points since all quantities involved admit a continuous limit.] Differentiating the scattering relation (2.12) and using (3.4) we then obtain the evolution equation for the scattering matrix $S$ :

$$
S_{t}=R_{+} S-S R_{-}, \quad k \in \Sigma^{\circ} .
$$

Substituting (3.4) into (3.6) yields the time evolution of the scattering coefficients. In particular, we have

$$
\begin{gathered}
s_{11}(k, t)=s_{11}(k, 0) \exp \left[i t\left(f_{+}(k)-f_{-}(k)\right)\right], \quad k \in \stackrel{\Sigma}{-}_{-}, \\
s_{21}(k, t)=s_{21}(k, 0) \exp \left[-i t\left(f_{+}(k)+f_{-}(k)\right]\right], \quad k \in \Sigma_{-}^{\circ} .
\end{gathered}
$$

As before, (3.7a) may be extended where $s_{11}(k, 0)$ is analytic, while (3.7b) can similarly be extended to $k \in \stackrel{\circ}{\Sigma}_{+}$ using (3.5) and the Wronskian definition (2.14) of $s_{21}(k, t)$. As a result, the reflection coefficient satisfies

$$
\rho(k, t)=\rho(k, 0) \exp \left[-2 i t f_{+}(k)\right], \quad k \in \Sigma_{+} .
$$


Moreover, (3.7a) also implies that, at the discrete eigenvalues,

$$
s_{11}^{\prime}\left(k_{j}, t\right)=s_{11}^{\prime}\left(k_{j}, 0\right) \exp \left[i t\left(f_{+}\left(k_{j}\right)-f_{-}\left(k_{j}\right)\right)\right], \quad j=1, \ldots, J,
$$

where again prime denotes differentiation with respect to $k$.

It now remains to determine the time evolution of the norming constants. To this end, note that (3.5) yields

$$
\left(\Phi_{-, 1}\right)_{t}=T \Phi_{-, 1}-i f_{-}(k) \Phi_{-, 1}, \quad\left(\Phi_{+, 2}\right)_{t}=T \Phi_{+, 2}+i f_{+}(k) \Phi_{+, 2}, \quad k \in \stackrel{\Sigma}{\Sigma}_{-} .
$$

Importantly, since all the terms involved admit analytic continuation off $\Sigma_{-}^{\circ}$, the above equations remain valid even for $k$ off $\Sigma_{-}^{\circ}$. Substituting these into (2.40) and simplifying gives the evolution of the $b_{j}(t)$ :

$$
b_{j}(t)=b_{j}(0) \exp \left[-i\left(f_{+}\left(k_{j}\right)+f_{-}\left(k_{j}\right)\right) t\right], \quad j=1, \ldots, J .
$$

Combining the previous equation with (3.9) gives the evolution of the norming constants:

$$
C_{j}(t)=C_{j}(0) \exp \left[-2 i f_{+}\left(k_{j}\right) t\right], \quad j=1, \ldots, J .
$$

We emphasize that, unlike the case of ZBC and symmetric NZBC, here $s_{11}(k, t)$ is not independent of time. Note also that $\operatorname{Im}\left[f_{ \pm}(k)\right] \neq 0$ for all $k \notin \Sigma_{-}$. This affects the behavior of $s_{11}(k, t)$ as $t \rightarrow \infty$. That is, for any fixed value of $k$ one may have sectors where $s_{11}(k, t) \rightarrow 0$ and others where $s_{11}(k, t) \rightarrow \infty$ as $t \rightarrow \infty$. It is also important to note, however, that the asymptotic behavior of $s_{11}(k, t)$ as $k \rightarrow \infty$ for any fixed value of $t$ is not affected by this time dependence. This is because

$$
\lambda_{ \pm}(k)=k-\frac{A_{ \pm}^{2}}{2 k}+O\left(1 / k^{3}\right), \quad k \rightarrow \infty
$$

which implies

$$
f_{+}(k)-f_{-}(k)=A_{+}^{2}-A_{-}^{2}+2 k\left(\lambda_{+}(k)-\lambda_{-}(k)\right)=O\left(1 / k^{2}\right), \quad k \rightarrow \infty .
$$

As a consequence, the normalization of the RHP (which is obtained by considering the limit $k \rightarrow \infty$ with $t$ fixed) is unaffected by the time dependence. Still, the limit as $t \rightarrow \infty$ with $k$ fixed and the limit as $k \rightarrow \infty$ with $t$ fixed do not commute, and one can expect that the non-uniformity of the behavior of $s_{11}(k, t)$ may affect the calculation of the long time asymptotics of the solutions.

\section{Inverse problem}

As usual, the inverse problem consists of reconstructing the eigenfunctions and the potential in terms of the scattering data (i.e., the reflection coefficient, the discrete spectrum, and the norming constants). We first formulate the inverse problem in terms of an open RHP. We then reduce the RHP to a standard set of linear integral equations (coupled to an algebraic system in the case of a non-empty discrete spectrum).

\subsection{Formulation of the Riemann-Hilbert problem}

The jump matrix for the RHP in the asymmetric case has a different expression in each of the regions that compose the jump, according to the cuts of $\lambda_{ \pm}$. More precisely, we need to distinguish the ranges $k \in \Sigma_{-}$and $k \in \Sigma_{o}$. The first of these ranges corresponds to the continuous spectrum of the scattering problem, allowing us to take advantage of the scattering relation in obtaining the jump matrix. Recalling the analyticity properties of the Jost eigenfunctions and scattering coefficients, we introduce the meromorphic matrix

$$
M(x, t, k)=\left(\frac{\mu_{-, 1}(x, t, k)}{s_{11}(k, t)}, \mu_{+, 2}(x, t, k)\right), \quad k \notin \Sigma_{+} .
$$

Note that, unlike the cases of ZBC and symmetric NZBC with a uniformization variable, the jumps of the RHP occur on an open contour. Therefore, in this case the meromorphic matrix has a unique representation over the whole cut plane. What is different are the projections of $M(x, t, k)$ to the cut from above/below. Specifically,

$$
M^{+}(x, t, k):=\lim _{\epsilon \downarrow 0} M(x, t, k+i \epsilon)=\left(\frac{\mu_{-, 1}(x, t, k)}{s_{11}(k, t)}, \mu_{+, 2}(x, t, k)\right), \quad k \in \Sigma_{+},
$$


whereas

$$
\begin{array}{ll}
M^{-}(x, t, k):=\lim _{\epsilon \uparrow 0} M(x, t, k+i \epsilon)=\left(\frac{\mu_{-, 1}^{-}(x, t, k)}{s_{11}^{-}(k, t)}, \mu_{+, 2}^{-}(x, t, k)\right), \quad k \in \Sigma_{-}, \\
M^{-}(x, t, k):=\lim _{\epsilon \uparrow 0} M(x, t, k+i \epsilon)=\left(\frac{\mu_{-, 1}(x, t, k)}{s_{11}^{-}(k, t)}, \mu_{+, 2}^{-}(x, t, k)\right), \quad k \in \Sigma_{o},
\end{array}
$$

where all of the above limits are assumed to be non-tangential, and where the continuity properties of the various columns of $\mu_{ \pm}(x, t, k)$ in the various ranges of $k$ follow trivially from those of $\Phi_{ \pm}(x, t, k)$. In other words, the projection of $M(x, t, k)$ from above is the same for both ranges of $k$, but the projection from below changes when moving from $\Sigma_{-}$to $\Sigma_{0}$ because $\mu_{-, 1}(x, t, k)$ is continuous across $\Sigma_{0}$, but not across $\Sigma_{-}$. Correspondingly, we need to write conditions that express the jump of $M(x, t, k)$ across the two ranges of $k$, namely:

$$
M^{+}(x, t, k)=M^{-}(x, t, k) V(x, t, k), \quad k \in \Sigma_{+},
$$

with

$$
V(x, t, k)= \begin{cases}V_{\Sigma_{-}}(x, t, k) & k \in \Sigma_{-} \\ V_{\Sigma_{o}}(x, t, k) & k \in \Sigma_{o}\end{cases}
$$

It should be clear from (4.1) and the properties of the eigenfunctions and scattering coefficients [discussed in Section 2.3] that on one hand $M(x, t, k)$ is continuous as $k \rightarrow \pm A_{-}$, but on the other hand $M(x, t, k)$ has a square root singularity as $k \rightarrow \pm A_{+}$. That is,

$$
\begin{gathered}
M(x, t, k)=O(1) \quad k \rightarrow \pm A_{-}, \\
M(x, t, k)=\left(O\left(k \mp A_{+}\right)^{1 / 2}, O(1)\right), \quad k \rightarrow \pm A_{+} .
\end{gathered}
$$

We next compute the jump matrices $V_{\Sigma_{-}}$and $V_{\Sigma_{0}}$ separately. Importantly, we will show that $V(x, t, k)$ is continuous both as $k \rightarrow \pm A_{-}$and as $k \rightarrow \pm A_{+}$.

Jump Matrix for $k \in \Sigma_{-}$. Recall that this range corresponds to the continuous spectrum of the scattering problem. Thus we begin by looking at (2.12) columnwise. What is not standard is that only half of the eigenfunctions appearing in these relations are analytic. We need to modify the above equations to obtain relations between the limits of the analytic Jost eigenfunctions from above and below the branch cut. Explicitly, we use (2.29) to express $\Phi_{-, 2}(x, t, k)$ and $\Phi_{+, 1}(x, t, k)$ in terms of $\tilde{\Phi}_{-, 1}(x, t, k)$ and $\tilde{\Phi}_{+, 2}(x, t, k)$, so that

$$
\begin{gathered}
\Phi_{-, 1}(x, t, k)=s_{11}(k, t) \frac{i q_{+}^{*}(t)}{k+\lambda_{+}(k)} \tilde{\Phi}_{+, 2}(x, t, k)+s_{21}(k, t) \Phi_{+, 2}(x, t, k), \quad k \in \Sigma_{-}, \\
-\frac{i q_{-}(t)}{k+\lambda_{-}(k)} \tilde{\Phi}_{-, 1}(x, t, k)=s_{12}(k, t) \frac{i q_{+}^{*}(t)}{k+\lambda_{+}(k)} \tilde{\Phi}_{+, 2}(x, t, k)+s_{22}(k, t) \Phi_{+, 2}(x, t, k), \quad k \in \Sigma_{-} .
\end{gathered}
$$

Rearranging, using (2.28) and putting in matrix form, we have

$$
\left(\frac{\Phi_{-, 1}(x, t, k)}{s_{11}(k, t)}, \Phi_{+, 2}(x, t, k)\right)=\left(\frac{\Phi_{-, 1}^{-}(x, t, k)}{s_{11}^{-}(k, t)}, \Phi_{+, 2}^{-}(x, t, k)\right) \frac{1}{i\left(k+\lambda_{+}(k)\right)} \sigma_{3} Q_{+}(t) V_{\rho}(k, t),
$$

where

$$
V_{\rho}(k, t)=\left(\begin{array}{cc}
1-|\rho(k, t)|^{2} & -\rho^{*}(k, t) \\
\rho(k, t) & 1
\end{array}\right), \quad k \in \Sigma_{-},
$$

and where we used the fact that $\bar{\rho}(k, t)=\rho^{*}(k, t)$ for $k \in \Sigma_{-}$[cf. (2.18)]. Writing the jump condition in terms of the modified eigenfunctions, we obtain (4.3) with (4.4) and

$$
V_{\Sigma_{-}}(x, t, k)=\left(E_{+}(k, t)-I\right)\left(\begin{array}{cc}
\mathrm{e}^{-i \lambda_{+}(k) x} & 0 \\
0 & \mathrm{e}^{i \lambda_{-}(k) x}
\end{array}\right) V_{\rho}(k, t)\left(\begin{array}{cc}
\mathrm{e}^{i \lambda_{-}(k) x} & 0 \\
0 & \mathrm{e}^{-i \lambda_{+}(k) x}
\end{array}\right),
$$

with $E_{+}(k, t)$ defined in (2.7), and where we used the fact that

$$
-i \sigma_{3} Q_{+}(t) /\left(k+\lambda_{+}\right)=E_{+}(k, t)-I .
$$


Jump matrix for $k \in \Sigma_{0}$. We wish to write $\Phi_{-, 1}(x, t, k) / s_{11}(k, t)$ and $\Phi_{+, 2}(x, t, k)$ in terms of $\Phi_{-, 1}(x, t, k) / s_{11}^{-}(k, t)$ and $\tilde{\Phi}_{+, 2}(x, t, k)$ (note that $\Phi_{-, 1}(x, t, k)$ is analytic across $\left.\Sigma_{0}\right)$. Using $(2.30 \mathrm{~b})$ gives

$$
\frac{\Phi_{-, 1}(x, t, k)}{s_{11}(k, t)}=\frac{\Phi_{-, 1}(x, t, k)}{s_{11}^{-}(k, t)} \frac{q_{+}(t)}{i\left(k+\lambda_{+}(k)\right)} \rho(k, t), \quad k \in \Sigma_{o}^{\circ} .
$$

To determine the other entries of the jump matrix, we use the first column of the scattering relation, (2.12), extended to $\Sigma_{0}$. Applying (2.29b) and rearranging, we again obtain (4.7), but where now $\Phi_{-, 1}^{-}(x, t, k)=\Phi_{-, 1}(x, t, k)$, and

$$
V_{\rho}(k, t)=\left(\begin{array}{cc}
0 & -1 / \rho(k, t) \\
\rho(k, t) & 1
\end{array}\right), \quad k \in \Sigma_{o} .
$$

As before, we rewrite the jump condition in terms of the modified eigenfunctions and consider the limits as $k$ to $\Sigma_{o}$ from below, obtaining (4.3) with

$$
V_{\Sigma_{o}}(x, t, k)=\left(E_{+}(k, t)-I\right)\left(\begin{array}{cc}
\mathrm{e}^{-i \lambda_{+}(k) x} & 0 \\
0 & \mathrm{e}^{-i \lambda_{-}(k) x}
\end{array}\right) V_{\rho}(k, t)\left(\begin{array}{cc}
\mathrm{e}^{i \lambda_{-}(k) x} & 0 \\
0 & \mathrm{e}^{-i \lambda_{+}(k) x}
\end{array}\right) .
$$

One could write an expression for $V_{\rho}(k, t)$ that is valid over the whole range of values of $k$ as

$$
V_{\rho}(k, t)=\left(\begin{array}{cc}
1-\rho(k, t) \bar{\rho}(k, t) & -\bar{\rho}(k, t) \\
\rho(k, t) & 1
\end{array}\right), \quad k \in \Sigma_{+},
$$

by formally defining

$$
\bar{\rho}(k, t)=1 / \rho(k, t), \quad k \in \Sigma_{o} .
$$

Again, recall the symmetry relation (2.18) for the reflection coefficients, namely $\bar{\rho}(k, t)=\rho^{*}(k, t)$ for $k \in \Sigma_{-}$. Equation (4.14) provides a relation that supplements (2.18) in the region $k \in \Sigma_{0}$. Moreover, since $\left|\rho\left( \pm A_{-}, t\right)\right|=1$ by (2.33), then at $k= \pm A_{-}$we have $\rho^{*}\left( \pm A_{-}, t\right)=1 / \rho\left( \pm A_{-}, t\right)$. Therefore, the extended $\bar{\rho}(k, t)$ thus defined is continuous at $k= \pm A_{-}$. In fact, recalling (2.36) we have that $\rho(k, t)$ and $\bar{\rho}(k, t)$ [and therefore $\left.V_{\rho}(k, t)\right]$ are continuous for all $k \in \Sigma_{+}$, including at the four branch points $k= \pm A_{ \pm}$.

Summarizing, the jump of $M(x, t, k)$ across $\Sigma_{+}$is given by (4.3), with $V_{\Sigma_{-}}(x, t, k)$ as in (4.9), and $V_{\Sigma_{o}}(x, t, k)$ as in (4.12), and with $V_{\rho}(k, t)$ given by (4.13). We can then write the RHP problem for $M(x, t, k)$ as

$$
M^{+}(x, t, k)=M^{-}(x, t, k)\left(E_{+}(k, t)-I\right)\left[I-V_{o}(x, t, k)\right], \quad k \in \Sigma_{+}
$$

where

$$
V_{o}(x, t, k)=\left\{\begin{array}{cc}
\left(\begin{array}{cc}
1-\mathrm{e}^{-i\left(\lambda_{+}(k)-\lambda_{-}(k)\right) x}\left(1-|\rho(k, t)|^{2}\right) & \mathrm{e}^{-2 i \lambda_{+}(k) x} \rho^{*}(k, t) \\
-\mathrm{e}^{2 i \lambda_{-}(k) x} \rho(k, t) & 1-\mathrm{e}^{-i\left(\lambda_{+}(k)-\lambda_{-}(k)\right) x}
\end{array}\right), & k \in \Sigma_{-}, \\
\left(\begin{array}{cc}
1 & \mathrm{e}^{-2 i \lambda_{+}(k) x} / \rho(k, t) \\
-\rho(k, t) & 1-\mathrm{e}^{-i\left(\lambda_{+}(k)+\lambda_{-}(k)\right) x}
\end{array}\right), & k \in \Sigma_{0} .
\end{array}\right.
$$

\subsection{Residue conditions, solution of the RHP and reconstruction formula}

To complete the formulation of the RHP, one must provide a normalization condition as $k \rightarrow \infty$, as well as residue conditions on the discrete spectrum. In the RHP for the case with ZBC, $M(x, t, k) \rightarrow I$ as $k \rightarrow \infty$. In that case, one subtracts both the contributions from the discrete spectrum and the asymptotic behavior and applies Cauchy projectors. In our case, however, the asympotic behaviors of $M(x, t, k)$ as $k \rightarrow \infty$ in the two half planes do not match. The main reason for this mismatch is that

$$
\lambda_{-}(k)=\left\{\begin{array}{l}
k-\frac{A_{-}^{2}}{2 k}+o(1 / k), \quad k \rightarrow \infty \wedge \operatorname{Im} k \geqslant 0, \\
-k+\frac{A_{-}^{2}}{2 k}+o(1 / k), \quad k \rightarrow \infty \wedge \operatorname{Im} k<0 .
\end{array}\right.
$$


As a result, (see Appendix D for details):

$$
M(x, t, k)= \begin{cases}I+O(1 / k), & k \rightarrow \infty \wedge \operatorname{Im} k>0, \\ -\frac{i}{k+\lambda_{+}(k)} \sigma_{3} Q_{+}(t)+O(1), & k \rightarrow \infty \wedge \operatorname{Im} k<0 .\end{cases}
$$

[Or equivalently $M(x, t, k)=E_{+}(k, t)+O(1)$ as $k \rightarrow \infty$ in the LHP, cf. (4.10).] To overcome this problem, it is convenient to first consider a toy RHP for a matrix $M_{*}(x, t, k)$ with the simpler jump condition

$$
M_{*}^{+}(x, t, k)=M_{*}^{-}(x, t, k)\left(E_{+}(k, t)-I\right), \quad k \in \Sigma_{+} .
$$

Equation (4.18) expresses the jump that one would obtain in the symmetric NZBC case with reflectionless potential. In this case, there is no "middle" region $\Sigma_{o}$. [See (A.19) for details.] By inspection, one sees that a solution to this problem is given by $M_{*}(x, t, k)=E_{+}(k, t)$, namely

$$
E_{+}(k, t)=E_{+}^{-}(k, t)\left(E_{+}(k, t)-I\right) .
$$

Moreover, the asymptotics of $M(x, t, k)$ given in (4.17) are the same as those of $E_{+}(k, t)$.

With these observations in mind, we perform a change of variable on our original RHP by introducing the new matrix $N(x, t, k)$ defined as

$$
M(x, t, k)=N(x, t, k) E_{+}(k, t) .
$$

Thus $N(x, t, k)=I+O(1 / k)$ as $k \rightarrow \infty$ in both the upper and lower half planes. The transformation (4.20) results in the new jump condition

$$
N^{+}(x, t, k)=N^{-}(x, t, k) \tilde{V}(x, t, k), \quad k \in \Sigma_{+},
$$

where $\tilde{V}(x, t, k)=E_{+}^{-}(k, t) V(x, t, k) E_{+}^{-1}(k, t)$ (recall that $E_{ \pm}^{+}(k, t)=E_{ \pm}(k, t)$ for all $\left.k \in \mathbb{R}\right)$. Taking into account (4.19), (4.3) and (4.15) we then find

$$
\tilde{V}(x, t, k)=E_{+}(k, t)\left(I-V_{o}(x, t, k)\right) E_{+}^{-1}(k, t), \quad k \in \Sigma_{+}
$$

where $V_{o}(x, t, k)$ is given by (4.15). Note that (4.5) imply $N(x, t, k)=O(1)$ as $k \rightarrow \pm A_{-}$and $N(x, t, k)=$ $\mathrm{O}\left(k \mp A_{+}\right)^{1 / 2}$ as $k \rightarrow \pm A_{+}$.

As mentioned above, we must also specify appropriate residue conditions which provide the contribution from the discrete spectrum. From (2.40) we have $\mu_{-, 1}\left(x, t, k_{j}\right)=b_{j}(t) \mu_{+, 2}\left(x, t, k_{j}\right) \mathrm{e}^{i\left(\lambda_{-, j}+\lambda_{+, j}\right) x}$ for $j=1, \ldots, J$. Since the zeros of $s_{11}(k, t)$ are simple (cf. Appendix E),

$$
\operatorname{Res}_{k=k_{j}}\left[\frac{\mu_{-, 1}(x, t, k)}{s_{11}(k, t)}\right]=\frac{\mu_{-, 1}\left(x, t, k_{j}\right)}{s_{11}^{\prime}\left(k_{j}, t\right)}=C_{j}(t) \mathrm{e}^{i\left(\lambda_{-, j}+\lambda_{+, j}\right) x} \mu_{+, 2}\left(x, t, k_{j}\right), \quad j=1, \ldots, J,
$$

where $C_{j}(t)$ is the norming constant associated with the eigenvalue $k_{j}$ and $\lambda_{ \pm, j}=\lambda_{ \pm}\left(k_{j}\right)$ [as defined in Section 2.4]. Thus,

$$
\operatorname{Res}_{k=k_{j}}[M(x, t, k)]=C_{j}(t) \mathrm{e}^{i\left(\lambda_{-, j}+\lambda_{+, j}\right) x}\left(M_{2}\left(x, t, k_{j}\right), 0\right), \quad j=1, \ldots, J .
$$

Moreover, since $E_{+}(k, t)$ is analytic for $k \notin \Sigma_{+}$and invertible for all $k \neq \pm A_{+}$, we have

$$
\operatorname{Res}_{k=k_{j}}[N(x, t, k)]=\operatorname{Res}_{k=k_{j}}[M(x, t, k)] E_{+}^{-1}\left(k_{j}, t\right)=C_{j}(t) \mathrm{e}^{i\left(\lambda_{-, j}+\lambda_{+, j}\right) x}\left(M_{2}\left(x, t, k_{j}\right), 0\right) E_{+}^{-1}\left(k_{j}, t\right), \quad j=1, \ldots, J .
$$

Explicitly, the residue conditions for $N(x, t, k)$ are given by (1.6).

To formally solve the RHP for $N(x, t, k)$, we subtract the asymptotic behavior as $k \rightarrow \infty$ and the residues obtained from the discrete spectrum; thus we consider the matrix $N(x, t, k)-I-\sum_{j=1}^{J} \operatorname{Res}_{k=k_{j}}[N(x, t, k)] /\left(k-k_{j}\right)$, which is analytic off $\Sigma_{+}$and goes to zero as $k \rightarrow \infty$ in both half planes. The solution of this RHP is then formally given by

$$
N(x, t, k)=I+\sum_{j=1}^{J} \frac{1}{k-k_{j}} \operatorname{Res}_{k=k_{j}}[N(x, t, k)]-\frac{1}{2 \pi i} \int_{\Sigma_{+}} \frac{\left[N^{-}(I-\tilde{V})\right](x, t, \zeta)}{\zeta-k} \mathrm{~d} \zeta, \quad k \in \mathbb{C} \backslash \Sigma_{+} .
$$


Recalling again (4.19), (4.20) and (4.22), straightforward algebra yields the following expression for $M(x, t, k)$ :

$$
\begin{aligned}
M(x, t, k)=E_{+}(k, t)+\sum_{j=1}^{J} \frac{1}{k-k_{j}} \operatorname{Res}_{k=k_{j}}[M(x, t, k)] E_{+}^{-1}\left(k_{j}, t\right) E_{+}(k, t) \\
\quad-\frac{1}{2 \pi i} \int_{\Sigma_{+}} \frac{\left[M^{-}\left(E_{+}-I\right) V_{o} E_{+}^{-1}\right](x, t, \zeta)}{\zeta-k} E_{+}(k, t) \mathrm{d} \zeta, \quad k \in \mathbb{C} \backslash \Sigma_{+},
\end{aligned}
$$

where (4.24) was used to express $\operatorname{Res}_{k=k_{j}} N(x, t, k)$ in terms of $\operatorname{Res}_{k=k_{j}} M(x, t, k)$. In order to close the system and to evaluate the reconstruction formula we need to supplement (4.26) with appropriate conditions for these residues. Even though the second column of $M(x, t, k)$ is analytic at $k=k_{j}$, the second column of $N(x, t, k)$ is not, since multiplication by $E_{+}(k, t)$ mixes the columns, as seen from (4.24). Thus, some care must be taken in evaluating the above expression. Nonetheless, taking into account (4.24) and evaluating the limit of the second column of (4.26) as $k \rightarrow k_{j}$ yields

$$
\begin{aligned}
& \left(1-\frac{i q_{+}}{2 \lambda_{+, j}^{2}} C_{j}(t) \mathrm{e}^{i\left(\lambda_{+, j}+\lambda_{-, j}\right) x}\right) M_{2}\left(x, t, k_{j}\right)=\left(I-\frac{1}{2 \pi i} \int_{\Sigma_{+}} \frac{\left[M^{-}\left(E_{+}-I\right) V_{o} E_{+}^{-1}\right](x, t, \zeta)}{\zeta-k_{j}} \mathrm{~d} \zeta\right. \\
& \left.\quad+\sum_{j^{\prime}=1}^{J} \frac{1}{k_{j}-k_{j^{\prime}}} C_{j^{\prime}}(t) \mathrm{e}^{i\left(\lambda_{+, j^{\prime}}+\lambda_{-, j^{\prime}}\right) x}\left(M_{2}\left(x, t, k_{j^{\prime}}\right), 0\right) E_{+}^{-1}\left(k_{j^{\prime}}, t\right)\right) E_{+, 2}\left(k_{j}, t\right), \quad j=1, \ldots, J,
\end{aligned}
$$

where the prime in the summation symbol denotes that the term $j^{\prime}=j$ is absent. As usual (4.26) and (4.27) [together with (4.24)] comprise a mixed linear system of algebraic-integral equations that provides the solution of the RHP.

We can now derive the reconstruction formula for the potential by looking at the asymptotic behavior of the solution of the RHP as $k \rightarrow \infty$. Indeed, the asymptotic behavior of the eigenfunctions computed from the direct problem [see Appendix D, specifically (D.2)], together with (4.1), yields

$$
q(x, t)=2 i \lim _{\substack{k \rightarrow \infty \\ \operatorname{Im} k>0}} k M_{12}(x, t, k) .
$$

Moreover, from (4.26) we have

$$
\begin{aligned}
M(x, t, k)=E_{+}(k, t) & +\frac{1}{k} \sum_{j=1}^{J} C_{j}(t) \mathrm{e}^{i\left(\lambda_{-, j}+\lambda_{+, j}\right) x}\left(M_{2}\left(x, t, k_{j}\right), 0\right) E_{+}^{-1}\left(k_{j}, t\right) \\
& +\frac{1}{2 \pi i k} \int_{\Sigma_{+}}\left[M^{-}\left(E_{+}-I\right) V_{o} E_{+}^{-1}\right](x, t, \zeta) \mathrm{d} \zeta+O\left(1 / k^{2}\right), \quad k \rightarrow \infty \wedge \operatorname{Im} k>0 .
\end{aligned}
$$

Computing the 1,2 component of (4.29) and using (4.28) then yields the reconstruction formula for the solution of the defocusing NLS equation with asymmetric NZBC:

$$
\begin{aligned}
& q(x, t)=q_{+}(t)\left(1-\sum_{j=1}^{J} \frac{C_{j}(t)}{\lambda_{+, j}} \mathrm{e}^{i\left(\lambda_{-, j}+\lambda_{+, j}\right) x} M_{12}\left(x, t, k_{j}\right)\right) \\
&+\frac{1}{2 \pi i} \int_{\Sigma_{-} \cup \Sigma_{o}} \frac{1}{\lambda_{+}(k)}\left[\left(\frac{i q_{+}(t)}{k+\lambda_{+}(k)} V_{o, 21}(x, t, k)+V_{o, 22}(x, t, k)\right) q_{+}(t) M_{11}^{-}(x, t, k)\right. \\
&\left.-\left(\frac{i q_{+}(t)}{k+\lambda_{+}(k)} V_{o, 11}(x, t, k)+V_{o, 12}(x, t, k)\right) q_{+}^{*}(t) M_{12}^{-}(x, t, k)\right] \mathrm{d} k,
\end{aligned}
$$

where the entries of $V_{o}(x, t, k)$ for $k \in \Sigma_{-}$and $k \in \Sigma_{o}$ are given by (4.15). 


\section{Discussion and final remarks}

As is always the case, the IST has been formulated under the assumption of existence (i.e., assuming the solution $q(x, t)$ of the IVP for the defocusing NLS equation with the given IC and asymmetric NZBC exists and satisfies the same regularity conditions as the IC). On the other hand, as usual one can then look at the representation formula as a definition of $q(x, t)$, and use it to prove that the function thus defined is the unique solution of the IVP in some appropriate functional space. The precise characterization of the admissible functional class, however, as well as that of the regularity properties of the scattering data and the issue of existence and uniqueness of the solution of the inverse problem, is left for future investigation. In this respect we should remark that this is a difficult problem even for the focusing NLS with ZBC [36, 37].

We should also mention that the formalism presented in this work could be extended in a relatively straightforward way to the case of $\mathrm{BC}$ which include non-zero and unequal asymptotic carriers, namely

$$
q(x, t)=A_{ \pm} \mathrm{e}^{i \alpha_{ \pm}-i \xi_{ \pm} x-i\left(\tilde{\xi}_{ \pm}^{2}+2 A_{ \pm}^{2}\right) t}+o(1), \quad x \rightarrow \pm \infty .
$$

The main difference in that case is that, when the NZBC (5.1) are given, the Jost eigenfunctions $\Phi_{ \pm}(x, t, k)$ are defined over $\Sigma_{ \pm}=\left(-\infty, \xi_{ \pm}-A_{ \pm}\right] \cup\left[\xi_{ \pm}+A_{ \pm}, \infty\right)$, respectively. It is then clear that, depending on the relative values of $A_{ \pm}$and $\xi_{ \pm}$, the ranges $\Sigma_{ \pm}$are not necessarily simply nested as in this work, but could also have more complicated overlap. Specifically, the "discrete spectrum" region $\mathbb{R} \backslash\left(\Sigma_{+} \cup \Sigma_{-}\right)$could be empty, while one could have a nonempty second "dispersive shock wave" region $\Sigma_{-} \backslash \Sigma_{+}$. The jump matrix in that case should be constructed using the opposite procedure as in the region $\Sigma_{o}$; namely, by expressing the analytic column of $\Phi_{+}$as a linear combination of the columns of $\Phi_{-}$and using the symmetries.

We expect that the IST formalism developed in this work will be instrumental for the calculation of the long-time asymptotic behavior of the solutions. Recall that, in the focusing case with ZBC, the problem was first studied in $[19,20]$ and then later revisited using the Deift-Zhou nonlinear steepest descent method [21, 22]. (See also [23] for the problem in the presence of real spectral singularities). In the case of symmetric NZBC, the long-time asymptotic behavior was first studied by [24], and was later revisited and made more rigorous in [25, 26] using the Deift-Zhou method. We should mention that the special case of a pure step IC was recently studied in [13], and that similar methods were used in [14] to study the short-time asymptotic behavior in the defocusing case with ZBC and discontinuous IC. For the focusing NLS equation with NZBC, the general theory of inverse scattering was developed in [27, 11], and the long-time asymptotics in the symmetric case and the case of one-sided step IC were studied respectively in [15] and [16]. We should also mention that the IST for the focusing NLS equation with the same kind of asymmetric NZBC considered in this work was recently studied in [28].

The results of this work also open up a number of interesting problems. Among them, one is the characterization of the scattering problem for specific classes of initial conditions, along the lines of what was done in [29] for the focusing NLS with ZBC, and in [30] and [6] for the focusing and defocusing cases with symmetric NZBC, respectively. Another interesting problem is whether there exist potentials with an infinite number of discrete eigenvalues, i.e., zeros for the analytic scattering coefficient, accumulating at one of the branch points. In this respect, recall that scattering problem for the Korteweg-deVries equation (which is the time-independent Schrödinger equation) always has a finite number of discrete eigenvalues, and that, for the defocusing NLS with symmetric NZBC, sufficient conditions can be formulated on the potential that guarantee that at most a finite number of zeros are present [7]. On the other hand, potentials with an infinite number of zeros are known to be allowed for the focusing NLS equation with ZBC [22].

A further problem is the generalization of these results to the Manakov system (i.e., the two-component vector NLS equation) with asymmetric NZBC. Here we note that, while the IST for the Manakov system with ZBC was formulated in 1974 [31], and can be generalized to an arbitrary number of components in a straightforward way [32], the case of NZBC is much more challenging, due to the defect of analyticity of some of the Jost eigenfunctions. The IST for the defocusing Manakov system with symmetric NZBC was finally formulated in [18] and rigorously revisited in [9] for the symmetric defocusing case, while the IST for the focusing Manakov system with NZBC was only recently formulated in [33]. On the other hand, the generalization to larger number of components in the case of NZBC is even more challenging due to the corresponding increase of the analyticity defect and the degeneracy of the scattering eigenvalues $[34,35]$. One could therefore expect that the development of the IST for coupled NLS systems with asymmetric NZBC will require significant further work. 
In conclusion, we should reiterate that even though in this work we have taken the branch cuts for the eigenvalues $\lambda_{ \pm}(k)$ along the half lines $\Sigma_{ \pm}=\left(-\infty,-A_{ \pm}\right] \cup\left[A_{ \pm}, \infty\right)$ (i.e., "outside"), one can equivalently formulate the IST when the branch cut for the eigenvalues is taken along the segments $\left[-A_{ \pm}, A_{ \pm}\right]$(i.e., "inside") instead. For comparison purposes, in Appendix B we present the formulation of the IST in the symmetric case with the branch cut inside. Each choice has its advantages and disadvantages. The analyticity properties of the Jost eigenfunctions are more standard when the branch cut inside, since half of the columns are analytic in the upper half plane and the other half in the lower half plane. This simplifies the formulation of the inverse problem. On the other hand, with the branch cut inside, the RHP acquires an additional jump along the segment $\left[-A_{ \pm}, A_{ \pm}\right]$. This introduces the further complication that the discrete eigenvalues are located along this jump. Thus, no choice seems to be clearly preferable with regard to the pure IST. At the same time, choosing the branch cut inside seems to be more convenient in the study of the long-time asymptotics [15, 13].

\section{Acknowledgments}

We thank Mark Ablowitz, David Trubatch, and Martin Klaus for insightful discussions related to this project, as well as the AIM for its hospitality during the SQuaRE program. We also acknowledge the anonymous reviewers, whose comments helped us improve the presentation of our work. This work was partially supported by the National Science Foundation under grants DMS-1311847 and DMS-1311883.

\section{Appendix A. IVP for symmetric NZBC without uniformization}

It is instructive to compare the formulation of the IST in the asymmetric case to the IST without uniformization in the case of symmetric NZBC, namely $A_{+}=A_{-}=q_{0} \neq 0$. In the symmetric case it is convenient to perform a change of variables to remove the time dependence of the $\mathrm{BC}$, which can be done by introducing the rescaled field $q(x, t)=\tilde{q}(x, t) \mathrm{e}^{-2 i q_{o}^{2} t}$ which satisfies a modified NLS equation

$$
i \tilde{q}_{t}+\tilde{q}_{x x}+2\left(q_{o}^{2}-|\tilde{q}|^{2}\right) \tilde{q}=0 .
$$

In what follows, we will omit the tildes as well as the term "modified" for brevity. Equation (A.1) is the compatibility condition of the Lax pair (2.1) where $T(x, t, k)$ is replaced by

$$
T(x, t, k)=-2 i k^{2} \sigma_{3}+i \sigma_{3} Q_{x}(x, t)+i\left(q_{o}^{2}-|q|^{2}(x, t)\right) \sigma_{3}+2 k Q(x, t) .
$$

The NZBC (1.3) then simplify to $q(x, t) \rightarrow q_{ \pm}$as $x \rightarrow \pm \infty$, with $q_{ \pm}$independent of time. (Also, without loss of generality we can take $q_{ \pm}=q_{o} \mathrm{e}^{ \pm i \alpha}$.) As a result, by defining the Jost solutions to be simultaneous solutions of both parts of the Lax pair, all of the scattering data will be time-independent. However, because of this change, the framework in this section is not merely a reduction $\lambda_{ \pm} \rightarrow \lambda$ and $\Sigma_{ \pm} \rightarrow \Sigma$. The formulation of the IST that follows can also be compared to the classical formulation of the IST for the case of symmetric NZBC, as presented in [8] (see also [6, 7]).

\section{A.1. Direct problem}

Jost eigenfunctions and scattering matrix. As $x \rightarrow \pm \infty$, the solutions of the Lax pair tend asymptotically to the solutions of

$$
\begin{gathered}
\Phi_{x}=X_{ \pm} \Phi, \quad \Phi_{t}=T_{ \pm} \Phi \\
X_{ \pm}=-i k \sigma_{3}+Q_{ \pm}, \quad T_{ \pm}=-2 i k^{2} \sigma_{3}+2 k Q_{ \pm} .
\end{gathered}
$$

The eigenvalues of the $x$-part and $t$-part are respectively $\pm i \lambda, \pm 2 i k \lambda$, where $\lambda^{2}=k^{2}-q_{o}^{2}$. As before, we take the branch cut on $\Sigma=\left(-\infty,-q_{0}\right] \cup\left[q_{0}, \infty\right)$, which coincides with the continuous spectrum. We define $\lambda$ as a singlevalued function of $k \in \mathbb{C}$, such that $\lambda(k)$ is continuous as $k$ approaches the branch cut from above. A convenient choice for the eigenvector matrices is

$$
E_{ \pm}(k)=I-\frac{i}{k+\lambda(k)} \sigma_{3} Q_{ \pm}
$$


where we emphasize that here $Q_{ \pm}$are independent of time. For all $k \in \Sigma$, one can then introduce two fundamental matrix solutions $\Phi_{ \pm}(x, t, k)$ to both parts of the Lax pair such that

$$
\Phi_{ \pm}(x, t, k)=E_{ \pm}(k) \mathrm{e}^{-i \theta(x, t, k) \sigma_{3}}(I+o(1)), \quad x \rightarrow \pm \infty,
$$

with

$$
\theta(x, t, k)=\lambda x+2 k \lambda t, \quad d(k)=1 / \operatorname{det} E_{ \pm}(k)=[k+\lambda(k)] /[2 \lambda(k)] .
$$

Also, we introduce the modified eigenfunctions as

$$
\mu_{ \pm}(x, t, k)=\Phi_{ \pm}(x, t, k) \mathrm{e}^{i \theta(x, t, k) \sigma_{3}} .
$$

As before, $\mu_{ \pm}(x, t, k)$ are rigorously defined in terms of integral equations:

$$
\begin{aligned}
& \mu_{-}(x, t, k)=E_{-}(k)+\int_{-\infty}^{x} E_{-}(k) \mathrm{e}^{-i \lambda(k)(x-y) \sigma_{3}} E_{-}^{-1}(k) \Delta Q_{-}(y, t) \mu_{-}(y, t, k) \mathrm{e}^{i \lambda(k)(x-y) \sigma_{3}} \mathrm{~d} y, \\
& \mu_{+}(x, t, k)=E_{+}(k)-\int_{x}^{\infty} E_{+}(k) \mathrm{e}^{-i \lambda(k)(x-y) \sigma_{3}} E_{+}^{-1}(k) \Delta Q_{+}(y, t) \mu_{+}(y, t, k) \mathrm{e}^{i \lambda(k)(x-y) \sigma_{3}} \mathrm{~d} y .
\end{aligned}
$$

where $\Delta Q_{ \pm}=Q-Q_{ \pm}$. Like in the case of asymmetric NZBC, if $(1+|\cdot|)\left(q(\cdot, t)-q_{ \pm}\right) \in L^{1}\left(\mathbb{R}^{ \pm}\right)$for all $t \in \mathbb{R}^{+}$, a Neumann series expansion for the above integral equations can be used to rigorously define the eigenfunctions and the analytic continuation of the appropriate columns off $\Sigma$, as well as to determine the asymptotics of the eigenfunctions as $k \rightarrow \infty$. In particular, (when the branch cut is outside), $\mu_{-, 1}$ and $\mu_{+, 2}$ are analytic in the whole complex plane except the branch cut.

Note that $\operatorname{det} \Phi_{ \pm}(x, t, k)=\operatorname{det} \mu_{ \pm}(x, t, k)=\operatorname{det} E_{ \pm}(k)=1 / d(k)$, independent of $x$ and $t$, which only vanishes when $\lambda=0$ (i.e., at the branch points $k= \pm q_{o}$ ). Thus, for all $k \in \mathbb{R} \backslash\left[-q_{o}, q_{o}\right], \Phi_{ \pm}(x, t, k)$ are fundamental matrix solutions of both parts of the Lax pair (2.1), and one can define the scattering matrix $S(k)$ by

$$
\Phi_{-}(x, t, k)=\Phi_{+}(x, t, k) S(k), \quad k \in \Sigma .
$$

Since $\Phi_{ \pm}(x, t, k)$ are simultaneous solutions of both parts of the Lax pair, the scattering matrix $S(k)$ is independent of both space and time. Moreover, $\operatorname{det} S(k)=1$. The entries of the scattering matrix may be written in terms of Wronskians as

$$
\begin{array}{ll}
s_{11}(k)=d(k) \operatorname{Wr}\left(\Phi_{-, 1}, \Phi_{+, 2}\right)(x, t, k), & s_{12}(k)=d(k) \operatorname{Wr}\left(\Phi_{-, 2}, \Phi_{+, 2}\right)(x, t, k), \\
s_{21}(k)=d(k) \operatorname{Wr}\left(\Phi_{+, 1}, \Phi_{-, 1}\right)(x, t, k), & s_{22}(k)=d(k) \operatorname{Wr}\left(\Phi_{+, 1}, \Phi_{-, 1}\right)(x, t, k) .
\end{array}
$$

The reflection coefficients appearing in the inverse problem are $\rho(k)=s_{21}(k) / s_{11}(k)$ and $\bar{\rho}(k)=s_{12}(k) / s_{22}(k)$. The scattering coefficients then inherit analyticity properties from the eigenfunctions via the Wronskian relations (A.8). In particular, $s_{11}(k)$ is analytic in $\mathbb{C} \backslash \Sigma$.

Symmetries. As in the case of ZBC and asymmetric NZBC, if $v(x, t, k)$ solves the scattering problem, then so does $\sigma_{1} v^{*}\left(x, t, k^{*}\right) \sigma_{1}$. For $k \in \Sigma, s_{11}(k)=s_{22}^{*}(k)$ and $s_{21}(k)=s_{12}^{*}(k)$. Thus, since $\operatorname{det} S(k)=1$, we have that $\left|s_{11}(k)\right|^{2} \geq 1$ on $\Sigma$, and thus $s_{11}(k)$ has no zeros there.

Moreover, for $k \notin \Sigma$, we have the following:

$$
\begin{gathered}
\sigma_{1} \Phi_{-, 1}^{*}\left(x, t, k^{*}\right)=\frac{-i q_{-}}{k-\lambda(k)} \Phi_{-, 1}(x, t, k), \quad \sigma_{1} \Phi_{+, 2}^{*}\left(x, t, k^{*}\right)=\frac{i q_{+}^{*}}{k-\lambda(k)} \Phi_{+, 2}(x, t, k), \\
s_{11}^{*}\left(k^{*}\right)=\left(q_{-} / q_{+}\right) s_{11}(k) .
\end{gathered}
$$

The above relations are derived using similar arguments as in the asymmetric case. 
To derive the second symmetry, for $k \in \Sigma$ we introduce another set of eigenfunctions as

$$
\tilde{\Phi}_{ \pm}(x, t, k)=\left[I-\frac{i}{k-\lambda(k)} \sigma_{3} Q_{ \pm}\right] \mathrm{e}^{i \theta(x, t, k) \sigma_{3}}(1+o(1)) \quad \text { as } x \rightarrow \pm \infty .
$$

We now have two fundamental matrix solutions to the Lax pair. Thus they are related by a matrix independent of $x$ and $t$, which we may determine by letting $x \rightarrow \pm \infty$ :

$$
\tilde{\Phi}_{ \pm}(x, t, k)=\Phi_{ \pm}(x, t, k)\left[-\frac{i}{k-\lambda(k)} \sigma_{3} Q_{ \pm}\right], \quad \Phi_{ \pm}(x, t, k)=\tilde{\Phi}_{ \pm}(x, t, k)\left[-\frac{i}{k+\lambda(k)} \sigma_{3} Q_{ \pm}\right], \quad k \in \Sigma
$$

Defining $\tilde{S}(k, \lambda(k))=S(k,-\lambda(k))$, we have

$$
s_{22}(k)=\left(q_{-} / q_{+}\right) \tilde{s}_{11}(k), \quad s_{21}(k)=-\left(q_{+}^{*} / q_{-}\right) \tilde{s}_{12}(k) .
$$

Limits of the eigenfunctions and scattering coefficients from below the branch cut. Recall $\lambda(k)$ is discontinuous across the branch cut, and specifically, $\lambda \mapsto-\lambda$ across $\Sigma$. $\Phi_{-, 1}(x, t, k)$ and $\Phi_{+, 2}(x, t, k)$ are analytic for $k \in \mathbb{C} \backslash \Sigma$ and are continuous to the branch cut from above. Then as $k \rightarrow \Sigma$ from above or below, we have

$$
\begin{array}{ll}
\Phi_{-, 1}^{-}(x, t, k):=\lim _{\epsilon \uparrow 0} \Phi_{-, 1}(x, t, k+i \epsilon)=\tilde{\Phi}_{-, 1}(x, t, k), & k \in \Sigma, \\
\Phi_{+, 2}^{-}(x, t, k):=\lim _{\epsilon \uparrow 0} \Phi_{+, 2}(x, t, k+i \epsilon)=\tilde{\Phi}_{+, 2}(x, t, k), & k \in \Sigma .
\end{array}
$$

We again use the tilde to denote both the alternate eigenfunctions and the limits of a function as it approaches the branch cut from below. Comparing with (A.11), we find

$$
\tilde{\Phi}_{-, 1}(x, t, k)=\frac{i}{k-\lambda} q_{-}^{*} \Phi_{-, 2}(x, t, k), \quad \tilde{\Phi}_{+, 2}(x, t, k)=-\frac{i}{k-\lambda} q_{+} \Phi_{+, 1}(x, t, k), \quad k \in \Sigma .
$$

Since $s_{11}(k)$ may be expressed as a Wronskian of functions which admit analytic continuation, we may determine its limit as $k \rightarrow \Sigma$ from below:

$$
s_{11}^{-}(k):=\lim _{\epsilon \uparrow 0} s_{11}(k+i \epsilon)=\frac{k-\lambda}{-2 \lambda} \operatorname{Wr}\left(\tilde{\Phi}_{-, 1}, \tilde{\Phi}_{+, 2}\right)=\frac{q_{+}}{q_{-}} s_{22}(k)=\tilde{s}_{11}(k), \quad k \in \Sigma .
$$

Finally, we observe that $\lim _{\epsilon \uparrow 0} \theta(k+i \epsilon)=-\theta(k)$, so

$$
\begin{aligned}
& \mu_{-, 1}^{-}(x, t, k)=\lim _{\epsilon \uparrow 0} \mu_{-, 1}(x, t, k+i \epsilon)=\tilde{\Phi}_{-, 1}(x, t, k) \mathrm{e}^{-i \theta(x, t, k)}, \\
& \mu_{+, 2}^{-}(x, t, k)=\lim _{\epsilon \uparrow 0} \mu_{+, 2}(x, t, k+i \epsilon)=\tilde{\Phi}_{+, 2}(x, t, k) \mathrm{e}^{i \theta(x, t, k)} .
\end{aligned}
$$

Note also that all eigenfunctions remain finite at the branch points but, for generic potentials, all scattering coefficients have square root singularities as $k \rightarrow \pm q_{o}$, and they all become proportional to each other in this limit.

Discrete eigenvalues. The discrete spectrum is the set of values $k_{j} \in \mathbb{C}$ for which $s_{11}\left(k_{j}\right)=0$. Since the scattering operator is self-adjoint, its eigenvalues lie on the real $k$-axis. On the other hand, the first symmetry implies $s_{11}(k)$ has no zeros for $k \in \Sigma$, so the discrete spectrum must be contained in the segment $\left(-q_{0}, q_{0}\right)$. The zeros of $s_{11}(k)$ are simple (see [8] and Appendix E), and we assume there are only a finite number $J$ of them; we write $\lambda_{j}$ for $\lambda\left(k_{j}\right)$. Using the Wronskian expressions (A.8), we may write

$$
\Phi_{-, 1}\left(x, t, k_{j}\right)=b_{j} \Phi_{+, 2}\left(x, t, k_{j}\right), \quad j=1, \ldots, J,
$$

for some constants $b_{j}$. The norming constants are $C_{j}=b_{j} /\left[s_{11}^{\prime}\left(k_{j}\right)\right]$ at each of the eigenvalues $k_{j}$. Starting from the relation (A.17), and using the properties of the first symmetry yields

$$
b_{j}=-\left(q_{+}^{*} / q_{-}\right) b_{j}^{*}, \quad\left[s_{11}^{\prime}\left(k_{j}\right)\right]^{*}=\left(q_{-} / q_{+}\right) s_{11}^{\prime}\left(k_{j}\right), \quad C_{j}^{*}=-\left(q_{+} / q_{+}^{*}\right) C_{j} .
$$




\section{A.2. Inverse problem}

As usual, we begin by looking at (A.7) columnwise. We then rewrite these equations to obtain a relation between the limits of the analytic Jost eigenfunctions and analytic scattering coefficients from above and below the branch cut (similarly to what was done for $k \in \Sigma_{-}$in the asymmetric NZBC). Explicitly, after rearranging terms, we obtain the matrix jump condition

$$
M^{+}(x, t, k)=M^{-}(x, t, k)\left(E_{+}(k)-I\right)\left[I-V_{o}(x, t, k)\right], \quad k \in \Sigma,
$$

where the analogue of (4.10) was used [namely, $E_{+}(k)-I=-i \sigma_{3} Q_{+} /(k+\lambda(k))$ ], with

$$
M(x, t, k)=\left(\frac{\mu_{-, 1}(x, t, k)}{s_{11}(k)}, \mu_{+, 2}(x, t, k)\right), \quad k \in \mathbb{C} \backslash \Sigma,
$$

implying $M^{+}(x, t, k)=M(x, t, k)$ and where $M^{-}(x, t, k)$ is obtained from (A.14), (A.15) and (A.16), and where

$$
V_{o}(x, t, k)=\mathrm{e}^{-i \theta(x, t, k) \sigma_{3}}\left(\begin{array}{cc}
|\rho(k)|^{2} & \bar{\rho}(k) \\
-\rho(k) & 0
\end{array}\right) \mathrm{e}^{i \theta(x, t, k) \sigma_{3}}, \quad k \in \Sigma .
$$

Similarly to the problem with asymmetric NZBC, the eigenfunctions are continuous as $k \rightarrow \pm q_{o}$ (under the same regularity assumptions for the potential), but the scattering coefficients [and therefore $M(x, t, k)$ ] have square root singularities in those limits. In particular,

$$
M(x, t, k)=\left(O\left(k \mp q_{0}\right)^{1 / 2}, O(1)\right), \quad k \rightarrow \pm q_{0} .
$$

On the other hand, the reflection coefficients [and therefore $V_{o}(x, t, k)$ ] remain finite as $k \rightarrow \pm q_{o}$, and in particular, $\left|\rho\left( \pm q_{0}\right)\right|=1$. As in the problem with asymmetric NZBC, the asympotics of $M(x, t, k)$ as $k \rightarrow \infty$ in each half plane do not match, since

$$
M(x, t, k)= \begin{cases}I+O(1 / k), & k \rightarrow \infty \wedge \operatorname{Im} k>0, \\ -\frac{i}{k+\lambda} \sigma_{3} Q_{+}+O(1), & k \rightarrow \infty \wedge \operatorname{Im} k<0 .\end{cases}
$$

To circumvent this problem, we again consider a toy RHP with the jump condition

$$
M_{o}^{+}(x, t, k)=M_{o}^{-}(x, t, k)[-i /(k+\lambda(k))] \sigma_{3} Q_{+},
$$

(which is the RHP obtained from a reflectionless potential with no discrete spectrum). By inspection, a solution to this problem is given by $M_{o}(x, t, k)=E_{+}(k)$. That is, recalling that $\lambda(k)$ [and therefore $E_{+}(k)$ ] is continuous from above the cut,

$$
E_{+}(k)=E_{+}^{-}(k)\left(E_{+}(k)-I\right), \quad k \in \Sigma .
$$

With this in mind, we define $N(x, t, k)$ as

$$
M(x, t, k)=N(x, t, k) E_{+}(k), \quad k \in \mathbb{C} \backslash \Sigma .
$$

Substituting into (A.19), we obtain the jump condition $N^{+}=N^{-} E_{+}^{-}\left(E_{+}-I\right)\left(I-V_{o}\right) E_{+}^{-1}$ for $k \in \Sigma$, or equivalently, using (A.23),

$$
N^{+}(x, t, k)=N^{-}(x, t, k)\left(I-E_{+}(k) V_{o}(x, t, k) E_{+}^{-1}(k)\right), \quad k \in \Sigma,
$$

together with the standard asymptotics

$$
N(x, t, k)=I+O(1 / k), \quad k \rightarrow \infty .
$$

Thus, the asymptotics in each half-plane now match, which will allow us to subtract the asymptotic behavior at infinity and apply Cauchy projectors as in the case of ZBC and symmetric NZBC. Before we do so, however, we need to consider the contribution from the discrete spectrum. From (A.17) we have

$$
\mu_{-, 1}\left(x, t, k_{j}\right)=b_{j} \mu_{+, 2}\left(x, t, k_{j}\right) \mathrm{e}^{2 i \theta\left(x, t, k_{j}\right)} .
$$


The zeros of $s_{11}(k)$ are simple, so

$$
\operatorname{Res}_{k=k_{j}}\left[\frac{\mu_{-, 1}(x, t, k)}{s_{11}(k)}\right]=\frac{\mu_{-, 1}\left(x, t, k_{j}\right)}{s_{11}^{\prime}\left(k_{j}\right)}=C_{j} \mu_{+, 2}\left(x, t, k_{j}\right) \mathrm{e}^{2 i \theta\left(x, t, k_{j}\right)},
$$

where $C_{j}$ is the norming constant (A.18) associated with eigenvalue $k_{j}$. Since $E_{+}(k)$ is analytic off the cut,

$$
\operatorname{Res}_{k=k_{j}}[M(x, t, k)]=\left(\frac{\mu_{-, 1}\left(x, t, k_{j}\right)}{s_{11}^{\prime}\left(k_{j}\right)}, 0\right)=\operatorname{Res}_{k=k_{j}}[N(x, t, k)] E_{+}\left(k_{j}\right) .
$$

Thus we consider $N(x, t, k)-I-\sum_{j=1}^{J} \operatorname{Res}_{k=k_{j}}[N(x, t, k)] /\left(k-k_{j}\right)$, which is analytic for $k \notin \Sigma$, and which goes to 0 as $k \rightarrow \infty$ in both half planes. After some algebra, the solution of this RHP is given by

$$
N(x, t, k)=I+\sum_{j=1}^{J} \frac{1}{k-k_{j}} \operatorname{Res}_{k=k_{j}}[N(x, t, k)]-\frac{1}{2 \pi i} \int_{\Sigma} \frac{\left(N^{-} E_{+} V_{o} E_{+}^{-1}\right)(x, t, \zeta)}{\zeta-k} \mathrm{~d} \zeta,
$$

and we have the solution of the original RHP (A.19)

$$
M(x, t, k)=\left[I+\sum_{j=1}^{J} \frac{1}{k-k_{j}} \operatorname{Res}_{k=k_{j}}[N(x, t, k)]-\frac{1}{2 \pi i} \int_{\Sigma} \frac{\left(M^{-}\left(E_{+}-I\right) V_{o} E_{+}^{-1}\right)(x, t, \zeta)}{\zeta-k} \mathrm{~d} \zeta\right] E_{+}(k) .
$$

Taking the limit of the second column as $k \rightarrow k_{j}$, we obtain the algebraic relations that complete the linear system for the solution of the RHP:

$$
\begin{aligned}
\left(1-\frac{i q_{+}}{2 \lambda_{j}^{2}} C_{j} \mathrm{e}^{2 i \theta\left(x, t, k_{j}\right)}\right) M_{2}\left(x, t, k_{j}\right) & =\left(I-\frac{1}{2 \pi i} \int_{\Sigma} \frac{\left[M^{-}\left(E_{+}-I\right) V_{o} E_{+}^{-1}\right](x, t, k)}{k-k_{j}} \mathrm{~d} k\right. \\
& \left.+\sum_{j^{\prime}=1}^{J} \frac{1}{k_{j}-k_{j^{\prime}}} C_{j^{\prime}} \mathrm{e}^{2 i \theta\left(x, t, k_{j}\right)}\left(M_{2}\left(x, t, k_{j^{\prime}}\right), 0\right) E_{+}^{-1}\left(k_{j^{\prime}}\right)\right) E_{+, 2}\left(k_{j}\right), \quad j=1, \ldots, J,
\end{aligned}
$$

where $\lambda_{j}=\lambda\left(k_{j}\right)$ for $j=1, \ldots, J$.

Reconstruction formula. As in the asymmetric case, $q(x, t)$ is obtained from $M(x, t, k)$ via (4.28). The asymptotic behavior of (A.26) as $k \rightarrow \infty$ in the UHP is

$$
\begin{aligned}
M(x, t, k)=E_{+}(k)+\frac{1}{k} \sum_{j=1}^{J} C_{j} \mathrm{e}^{2 i \theta\left(x, t, k_{j}\right)}\left(M_{2}\left(x, t, k_{j}\right), 0\right) & E_{+}^{-1}\left(k_{j}\right) \\
& +\frac{1}{2 \pi i k} \int_{\Sigma}\left[M^{-}\left(E_{+}-I\right) V_{o} E_{+}^{-1}\right](x, t, \zeta) \mathrm{d} \zeta+O\left(1 / k^{2}\right) .
\end{aligned}
$$

Computing the 1,2 component of the above expression [using similar steps as in the asymmetric case] then yields the reconstruction formula for the solution of the defocusing NLS equation:

$$
\begin{array}{r}
q(x, t)=q_{+}\left(1-\sum_{j=1}^{J} \frac{C_{j}}{\lambda_{j}} \mathrm{e}^{2 i \theta\left(x, t, k_{j}\right)} M_{12}\left(x, t, k_{j}\right)\right)+\frac{1}{2 \pi i} \int_{\Sigma} \frac{1}{\lambda(k)}\left[\frac{i q_{+}^{2}}{k+\lambda(k)} V_{o, 21}(x, t, k) M_{11}^{-}(x, t, k)\right. \\
\left.-q_{+}^{*}\left(\frac{i q_{+}}{k+\lambda(k)} V_{o, 11}(x, t, k)+V_{o, 12}(x, t, k)\right) M_{12}^{-}(x, t, k)\right] \mathrm{d} k .
\end{array}
$$

Alternatively, $q(x, t)$ can be expressed in terms of the Jost eigenfunctions. From the definition of $M(x, t, k)$, the limits from below the cut and the symmetries one can check that

$$
M^{-}(x, t, k)=\left(i(k+\lambda) \mu_{-, 2}(x, t, k) /\left(q_{+}(t) s_{22}(k, t)\right), i q_{+}(t) \mu_{+, 1}(x, t, k) /(k-\lambda)\right), \quad k \in \Sigma .
$$


Also, (A.5) and the scattering relation (A.7) imply

$$
\mu_{-, 1}(x, t, k)=s_{12}(k, t) \mu_{+, 1}(x, t, k)+e^{-2 i \theta(x, t, k)} s_{2,2}(k, t) \mu_{+, 2}(k, t), \quad k \in \Sigma .
$$

Straightforward algebra then yields:

$$
\begin{aligned}
q(x, t)= & q_{+}-q_{+} \sum_{j=1}^{J} \frac{C_{j}}{\lambda_{j}} \mu_{+, 12}\left(x, t, k_{j}\right) \mathrm{e}^{2 i \theta\left(x, t, k_{j}\right)} \\
& +\frac{1}{2 \pi} \int_{\Sigma} \frac{1}{\lambda(k)}\left[\mathrm{e}^{-2 i \theta(x, t, k)}(k+\lambda(k)) \bar{\rho}(k) \mu_{+, 11}(x, t, k)-i q_{+} \mathrm{e}^{2 i \theta(x, t, k)} \rho(k) \mu_{+, 12}(x, t, k)\right] \mathrm{d} k .
\end{aligned}
$$

\section{A.3. Comparison between the reconstruction formulae with and without uniformization}

We next show how the reconstruction formula (A.31) obtained from the IST formulated without the use of a uniformization variable agrees with the reconstruction formula derived using the uniformization variable. We first discuss the case when no discrete eigenvalues are present, in which case the reconstruction formula obtained using the uniformization variable is $[6,8]$

$$
q(x, t)=q_{+}+\frac{1}{2 \pi} \int_{\mathbb{R}} \mathrm{e}^{-2 i \theta(x, t, z)} \bar{\rho}(z) \mu_{+, 11}(x, t, z) \mathrm{d} z,
$$

where the uniformization variable is $z=k+\lambda(k)$. [Recall that the transformation $k \mapsto z$ is inverted by $k=$ $\frac{1}{2}\left(z+q_{o}^{2}\right) / z$ and $\lambda=\frac{1}{2}\left(z-q_{o}^{2} / z\right)$.] Breaking the integral (A.32) into an integral over $\Sigma$ and one over $\mathbb{R} \backslash \Sigma$, we see that

$$
\int_{\Sigma} \mathrm{e}^{-2 i \theta(x, t, z)} \bar{\rho}(z) \mu_{+, 11}(x, t, z) \mathrm{d} z=\int_{\Sigma} \mathrm{e}^{-2 i \theta(x, t, k)} \bar{\rho}(k) \frac{k+\lambda(k)}{\lambda(k)} \mu_{+, 11}(x, t, k) \mathrm{d} k,
$$

where we made the change of variables $z=k+\lambda(k)$ and with some abuse of notation we write $\rho(k)$ for $\rho(z(k))$, $\theta(x, t, k)$ for $\theta(x, t, z(k))$ etc., and viceversa. The range $z \in\left[-q_{0}, q_{0}\right]$ can also be obtained by taking $k \in \Sigma$, but with the opposite sign for $\lambda(k)$ and the opposite orientation for the contour. In other words, the appropriate change of variables in this case is $z=k-\lambda(k)$, and some care must be taken in evaluating the integrand. In particular, dropping the explicit dependence on $k$, and again with some abuse of notation, using the symmetries of the problem we have:

$$
\mu_{+, 11}(x, t, k,-\lambda)=\frac{i q_{+}^{*}}{k-\lambda} \mu_{+, 12}(x, t, k, \lambda), \quad \bar{\rho}(k,-\lambda)=-\frac{q_{+}}{q_{+}^{*}} \rho(k, \lambda), \quad \mathrm{e}^{-2 i \theta(x, t, k,-\lambda)}=\mathrm{e}^{2 i \theta(x, t, k, \lambda)} .
$$

Thus

$$
\int_{\mathbb{R} \backslash \Sigma} \bar{\rho}(z) \mathrm{e}^{-2 i \theta(x, t, z)} \mu_{+, 11}(x, t, z) \mathrm{d} z=-\int_{\Sigma} \frac{i q_{+}}{\lambda(k)} \rho(k) \mathrm{e}^{2 i \theta(x, t, k)} \mu_{+, 12}(x, t, k) \mathrm{d} k .
$$

Combining (A.33) and (A.34) then confirms that indeed (A.32) coincides with (A.31).

One can also check that the formulations of the problem with and without uniformization are equivalent by deriving the one-soliton solution from the IST without uniformization. Considering the reflectionless case [i.e., $\rho(k)=0$ for all $k \in \Sigma$ ] with just one discrete eigenvalue at $k=k_{1}$, we may use (A.27) to solve for $\mu_{+, 12}\left(x, t, k_{1}\right)$

$$
\mu_{+, 12}\left(x, t, k_{1}\right)=-\frac{i q_{+}}{k_{1}+\lambda_{1}} /\left(1-\frac{i q_{+}}{2 \lambda_{1}} C_{1} \mathrm{e}^{2 i \theta\left(k_{1}\right)}\right) .
$$

When formulated for the uniformization variable, the norming constant may be written as

$$
C_{\text {unif }}=\frac{2 i \lambda_{1}\left(k_{1}+\lambda_{1}\right)}{q_{+}} \mathrm{e}^{-2 i \lambda_{1} \zeta_{1}},
$$


with $\zeta_{1} \in \mathbb{R}$. Then

$$
C_{1}=C_{\text {unif }} / \frac{d z}{d k}\left(k_{1}\right)=C_{\text {unif }} \frac{\lambda_{1}}{k_{1}+\lambda_{1}}=\frac{2 i \lambda_{1}^{2}}{q_{+}} \mathrm{e}^{-2 i \lambda_{1} \zeta_{1}} .
$$

The reconstruction formula becomes

$$
q(x, t)=q_{+}-\frac{2 q_{+} \lambda_{1}}{k_{1}+\lambda_{1}} /\left(\mathrm{e}^{-2 i \theta\left(k_{1}\right)+2 i \lambda_{1} \zeta_{1}}+1\right) .
$$

Setting $k_{1}=\cos \alpha, \lambda_{1}=i \sin \alpha$, we recover the familiar dark soliton solution of the defocusing NLS equation with symmetric NZBC, namely:

$$
q(x, t)=q_{+} \mathrm{e}^{-i \alpha}\left(\cos \alpha+i \sin \alpha \tanh \left[\left|\lambda_{1}\right|\left(x-\zeta_{1}+2 k_{1} t\right)\right]\right) .
$$

\section{Appendix B. IST for symmetric NZBC: branch cut inside}

We next show how the IST can equivalently be formulated when the branch cut for the eigenvalues $\lambda_{ \pm}(k)$ is taken along the segments $\left[-A_{ \pm}, A_{ \pm}\right]$instead of along the half lines $\Sigma_{ \pm}=\left(-\infty,-A_{ \pm}\right] \cup\left[A_{ \pm}, \infty\right)$. For brevity, we present the IST only in the case of symmetric NZBC (i.e., the limit $A_{+}=A_{-}=q_{0}$ ), but it should be clear that the formalism generalizes to asymmetric NZBC. Indeed, as a further example in Appendix C we present the IST in the opposite limit, namely $A_{+}=0$, corresponding to one-sided NZBC.

Direct problem. As in the case with the branch cut outside, we define $\lambda(k)$ on the branch cut $\left[-q_{0}, q_{0}\right]$ to be continuous from above. The continuous spectrum is still the set $\Sigma=\left(-\infty,-q_{0}\right] \cup\left[q_{0}, \infty\right)$. Namely for all $k \in \Sigma$, we can define the Jost solutions $\Phi_{ \pm}(x, t, k)$ and the modified eigenfunctions $\mu_{ \pm}(x, t, k)$, using the same relations as in Appendix A. [That is, $\mu_{ \pm}(x, t, k)$ again satisfy (A.6).] For all $k \in \stackrel{\Sigma}{\Sigma}$, we can also define the scattering matrix $S(k)$ via (A.7). The analyticity properties of the eigenfunctions and scattering coefficients, however, are very different from the case with the branch cut outside. Namely, using the same integral equations (A.6), one can show that now $\mu_{-, 1}(x, t, k)$ and $\mu_{+, 2}(x, t, k)$ are analytic in the UHP, while $\mu_{-, 2}(x, t, k)$ and $\mu_{+, 1}(x, t, k)$ are analytic in the LHP, exactly as in the problem with ZBC, and unlike the problem with the branch cut outside. Since the scattering coefficients may be defined using the Wronskian relations, $s_{11}(k)$ may be analytically continued to the UHP, and $s_{22}(k)$ to the LHP, again as in the problem with ZBC, and unlike the problem with the branch cut outside. The difference from the case of ZBC is of course the presence of the branch cut $\left[-q_{0}, q_{0}\right]$, and the fact that the continuous spectrum is only the subset $\Sigma$ of the real $k$-axis.

Symmetries. If $v(x, t, k)$ solves the first of (2.1), then so does $\hat{v}(x, t, k)=\sigma_{1} v^{*}\left(x, t, k^{*}\right) \sigma_{1}$. Comparing asymptotics at $x= \pm \infty$, we have that

$$
\Phi_{ \pm}(x, t, k)=\sigma_{1} \Phi_{ \pm}^{*}(x, t, k) \sigma_{1}, \quad k \in \Sigma .
$$

Importantly, since all of the columns of $\Phi_{ \pm}(x, t, k)$ are analytic, each column of the above relation can also be extended to $k \notin \Sigma$ [and in particular evaluated for $k \in\left(-q_{0}, q_{0}\right)$ ] by considering the Schwarz extension of each column. Substituting into (A.7), we have

$$
S(k)=\sigma_{1} S^{*}(k) \sigma_{1}, \quad k \in \Sigma,
$$

which implies

$$
s_{11}(k)=s_{22}^{*}\left(k^{*}\right), \quad \operatorname{Im} k \geqslant 0, \quad s_{12}(k)=s_{21}^{*}(k), \quad k \in \Sigma,
$$

where the symmetry for the analytic scattering coefficients was extended via the Schwarz reflection principle. For the second symmetry, we again define two new matrix solutions $\tilde{\Phi}_{ \pm}(x, t, k)$ as in (A.10), which again satisfy (A.11), and the scattering matrix $\tilde{S}(k)$, which satisfies (A.12). In particular, the symmetries (A.11) of the eigenfunctions yield

$$
\tilde{\Phi}_{+, 1}(x, t, k)=\frac{i q_{+}^{*}}{k-\lambda} \Phi_{+, 2}(x, t, k), \quad \tilde{\Phi}_{-, 2}(x, t, k)=-\frac{i q_{-}}{k-\lambda} \Phi_{-, 1}(x, t, k), \quad k \in\left(-q_{0}, q_{0}\right) .
$$

In turn, these imply $\tilde{s}_{22}(k)=s_{11}(k)\left(q_{-} / q_{+}\right)$for all $k \in\left(-q_{0}, q_{0}\right)$. 
Limits of the eigenfunctions and scattering coefficients from below the branch cut. The limits of $\Phi_{+, 1}, \Phi_{-, 2}$ as they approach the branch cut from below are

$$
\begin{array}{ll}
\Phi_{+, 1}^{-}(x, t, k):=\lim _{\epsilon \uparrow 0} \Phi_{+, 1}(x, t, k+i \epsilon)=\tilde{\Phi}_{+, 1}(x, t, k), & k \in\left(-q_{0}, q_{0}\right), \\
\Phi_{-, 2}^{-}(x, t, k):=\lim _{\epsilon \uparrow 0} \Phi_{-, 2}(x, t, k+i \epsilon)=\tilde{\Phi}_{-, 2}(x, t, k), & k \in\left(-q_{0}, q_{0}\right) .
\end{array}
$$

The limit of $s_{22}(k)$ as $k$ approaches the branch cut from below is

$$
s_{22}^{-}(k):=\lim _{\epsilon \uparrow 0} s_{22}(k+i \epsilon)=-\operatorname{Wr}\left(\tilde{\Phi}_{+, 1}(x, t, k), \tilde{\Phi}_{-, 2}(x, t, k)\right) \frac{k-\lambda(k)}{2 \lambda(k)}=\frac{q_{-}}{q_{+}} s_{11}(k), \quad k \in\left(-q_{0}, q_{0}\right),
$$

Taking into account that $\lim _{\epsilon \uparrow 0} \theta(x, t, k+i \epsilon)=-\theta(x, t, k)$ for $k \in\left(-q_{0}, q_{0}\right)$ and the definition (A.5) of the modified eigenfunctions, and in preparation for the inverse problem, we combine the limits to the branch cut from below of the functions analytic in the LHP into the matrix expression

$$
\left(\frac{\mu_{-, 1}(x, t, k)}{s_{11}(k)}, \mu_{+, 2}(x, t, k)\right)=\left(\mu_{+, 1}^{-}(x, t, k), \frac{\mu_{-, 2}^{-}(x, t, k)}{s_{22}^{-}(k)}\right) \frac{-i}{k+\lambda(k)} \sigma_{3} Q_{+}, \quad k \in\left(-q_{0}, q_{0}\right) .
$$

Importantly, the combination of the two symmetries of the analytic scattering coefficients results in a constraint on the values of $s_{11}(k)$. Indeed, noting that $\tilde{s}_{22}(k)=s_{22}^{-}(k)$, we have

$$
s_{11}^{*}(k)=s_{11}(k) \frac{q_{-}}{q_{+}}, \quad k \in\left(-q_{0}, q_{0}\right) .
$$

Thus (B.4) implies that for $k \in\left(-q_{0}, q_{0}\right)$, we have $\arg s_{11}(k)=\arg q_{+}$or $\arg s_{11}(k)=\arg q_{+}+\pi$. This relation will result in a constraint on the norming constants.

Discrete eigenvalues. The number and location of discrete eigenvalues in the case of symmetric NZBC were discussed in Appendix A. At any such value $k_{j} \in\left(-q_{0}, q_{0}\right)$ for which $s_{11}\left(k_{j}\right)=0$ for $j=1, \ldots, J,($ A.17) holds. Moreover, using the symmetries (B.1) of the scattering coefficients, we know that zeros of $s_{11}(k)$ are also zeros of $s_{22}(k)$. Combining (B.1) with (A.17) we then have

$$
\tilde{\Phi}_{-, 2}\left(x, t, k_{j}\right)=-\frac{q_{+}^{*}}{q_{-}} b_{j} \tilde{\Phi}_{+, 1}\left(x, t, k_{j}\right), \quad j=1, \ldots, J .
$$

This relation will be needed in order to subtract the residues from the RHP.

We will also need to relate $s_{11}^{\prime}\left(k_{j}\right)$ to $s_{22}^{\prime}\left(k_{j}\right)$, where the derivatives are taken in upper and lower half neighborhoods of $k_{j}$, respectively. Indeed, when the branch cut is taken inside, the zeros of $s_{11}(k)$ and $s_{22}(k)$ lie on the boundary of their domains of analyticity. On the other hand, one can show that, just like the condition $q(x, t)-q_{ \pm} \in$ $L^{1}\left(\mathbb{R}^{ \pm}\right)$guarantees analyticity of $s_{11}(k)$ and $s_{22}(k)$ in $\mathbb{C}^{ \pm}$as well as continuity up to the real axis away from the branch points, the condition $(1+|x|)\left(q(x, t)-q_{ \pm}\right) \in L^{1}\left(\mathbb{R}^{ \pm}\right)$[which was already required in order to the eigenfunctions are well defined at the branch points] guarantees that the derivatives of $s_{11}(k)$ and $s_{22}(k)$ are also continuous up to the boundary, away from the branch points. Equation (B.1) then implies

$$
s_{22}^{\prime}\left(k_{j}\right)=\left[s_{11}^{\prime}\left(k_{j}\right)\right]^{*}, \quad j=1, \ldots, J .
$$

Moreover, since the complex derivative is independent of direction, we can also differentiate $s_{22}(k)$ along the real $k$-axis. Using the relation (B.2), we obtain

$$
s_{22}^{\prime}\left(k_{j}\right)=\frac{q_{-}}{q_{+}} s_{11}^{\prime}\left(k_{j}\right), \quad j=1, \ldots, J
$$

Using (B.5), we may write

$$
\frac{\tilde{\mu}_{-, 2}\left(k_{j}\right)}{s_{22}^{\prime}\left(k_{j}\right)}=-\frac{i q_{+}}{k_{j}-\lambda_{j}} \frac{\mu_{-, 1}\left(k_{j}\right)}{s_{11}^{\prime}\left(k_{j}\right)}, \quad j=1, \ldots, J,
$$

which is the relation that we will need to regularize the RHP. 
Inverse problem. We now formulate the RHP, and show that the problem reduces to the RHP that we derived when the branch cut was taken outside. For $k \in \Sigma$, the Jost eigenfunctions are related to each other by the scattering matrix via (A.7). We therefore define

$$
M(x, t, k)= \begin{cases}\left(\frac{\mu_{-, 1}(x, t, k)}{s_{11}(k)}, \mu_{+, 2}(x, t, k)\right), & \operatorname{Im} k>0, \\ \left(\mu_{+, 1}(x, t, k), \frac{\mu_{-, 2}(x, t, k)}{s_{22}(k)}\right), & \operatorname{Im} k<0 .\end{cases}
$$

Using the scattering relation (A.7), it is then straightforward to show that

$$
M^{+}(x, t, k)=M^{-}(x, t, k)\left(I-V_{o}(x, t, k)\right), \quad k \in \Sigma,
$$

where $V_{o}(x, t, k)$ has the same expression as in (A.21). (Indeed, the expression for the jump matrix in this range of values of $k$ is exactly the same as in the case of ZBC, regardless of whether the branch cut is taken outside or inside.) Now, however, we must take into account the jump of $M(x, t, k)$ across the branch cut, which is given by (B.3). Thus we may write

$$
M^{+}(x, t, k)=M^{-}(x, t, k) V(x, t, k), \quad k \in \mathbb{R},
$$

where

$$
V(x, t, k)= \begin{cases}I-V_{o}(x, t, k), & k \in \Sigma, \\ E_{+}(k)-I, & k \in\left(-q_{o}, q_{o}\right) .\end{cases}
$$

We may subtract off the residues using the relationship (B.6). Then, as in the case with the branch cut outside, we use a rescaling, defining $N(x, t, k)$ as in (A.24). This gives the new jump condition $N^{+}(x, t, k)=N^{-}(x, t, k) \tilde{V}(x, t, k)$ where

$$
\tilde{V}(x, t, k)= \begin{cases}I-E_{+}(k) V_{o}(x, t, k) E_{+}^{-1}(k), & k \in \Sigma, \\ I, & k \in\left(-q_{0}, q_{o}\right) .\end{cases}
$$

This, however, is precisely the RHP obtained by taking the branch cut outside, which is what we would expect. (Note that the two values of $\lambda(k)$ obtained with the different choice of branch cut agree on the real axis — in fact, they agree on the closure of the upper half plane.)

\section{Appendix C. IST for the defocusing NLS equation with one-sided NZBC}

As a further application of the methods presented in the main text, here we consider the IVP for the defocusing NLS equation (A.1) with the following one-sided NZBC:

$$
\lim _{x \rightarrow-\infty} q(x, t)=q_{0}, \quad \lim _{x \rightarrow \infty} q(x, t)=0,
$$

with $q_{0}>0$, and where without loss of generality we have set the asymptotic phase to zero using the phase invariance of the NLS equation. Of course one could also use the scaling invariance of the NLS equation to set $q_{0}=1$, but we will not do so, as we want to allow for the possibility of taking the limit $q_{0} \rightarrow 0$. As in the case of symmetric NZBC, the extra term proportional to $q_{0}$ in (A.1) was added so that the BC (C.1) as $x \rightarrow-\infty$ is independent of time, which also makes it possible to introduce the Jost solutions as simultaneous solutions of both parts of the Lax pair.

The Lax pair for the above defocusing NLS equation (A.1) is still given by (2.1) with $T(x, t, k)$ replaced by (A.2). As a special case, we will consider the following step IC:

$$
q(x, 0)= \begin{cases}q_{0}, & x<0 \\ 0, & x>0\end{cases}
$$

We should mention that the $\mathrm{BC}$ (C.1) also allows the introduction of a uniformization variable, similarly to the case of symmetric NZBC [8] and unlike the case of asymmetric NZBC. 


\section{C.1. Direct problem}

Jost eigenfunctions. As usual, the Jost eigenfunctions $\Phi_{ \pm}(x, t, k)$ are the solutions of (2.1) with prescribed asymptotic behavior as $x \rightarrow \pm \infty$. We first discuss the limit $x \rightarrow \infty$. Since $q(x, t) \rightarrow 0$ as $x \rightarrow \infty$, we have $\lim _{x \rightarrow \infty} X(x, t, k)=$ $X_{+}(k)=-i k \sigma_{3}$ and $\lim _{x \rightarrow \infty} T(x, t, k)=T_{+}(k)=-\left(2 k^{2}-q_{o}^{2}\right) \sigma_{3}$. We can then define $\Phi_{+}(x, t, k)$ as the matrix solution of both parts of the Lax pair such that

$$
\Phi_{+}(x, t, k)=\mathrm{e}^{-i \theta_{+}(x, t, k) \sigma_{3}}+o(1), \quad x \rightarrow \infty,
$$

where

$$
\theta_{+}(x, t, k)=k x+\left(2 k^{2}-q_{o}^{2}\right) t .
$$

Obviously $\Phi_{+}(x, t, k)$ is well-defined $\forall k \in \mathbb{R}$. Moreover, using standard arguments one can show that its columns $\Phi_{+, 1}(x, t, k)$ and $\Phi_{+, 2}(x, t, k)$ admit analytic continuation to $\operatorname{Im} k<0$ and $\operatorname{Im} k>0$, respectively.

The limit $x \rightarrow-\infty$ is slightly more involved, since $\lim _{x \rightarrow-\infty} X(x, t, k)=X_{-}(k)=-i k \sigma_{3}+Q_{-}$and $\lim _{x \rightarrow-\infty} T(x, t, k)=T_{-}(k)=2 k X_{-}(k)$, where $Q_{-}=\lim _{x \rightarrow-\infty} Q(x, t)$ as before. The eigenvalue-eigenvector relations for $X_{-}$and $T_{-}$are

$$
X_{-} E_{-}=E_{-}\left(-i \lambda \sigma_{3}\right), \quad T_{-} E_{-}=E_{-}\left(-2 i k \lambda \sigma_{3}\right)
$$

where $\lambda^{2}=k^{2}-q_{o}^{2}$ and

Note, for future convenience, that

$$
E_{-}(k)=I-\frac{i}{k+\lambda} \sigma_{3} Q_{-}
$$

$$
\operatorname{det} E_{-}(k)=\frac{2 \lambda}{k+\lambda}=1-\frac{q_{o}^{2}}{(k+\lambda)^{2}}=: 1 / d_{-}(k), \quad E_{-}^{-1}(k)=d_{-}(k)\left[I+\frac{i}{k+\lambda} \sigma_{3} Q_{-}\right] .
$$

As before, $\lambda(k)$ is a multi-valued function with branching. Here we will take the branch cut to be the real line segment $\left[-q_{0}, q_{0}\right]$, we take the principal branch of $\lambda(k)$, such that $\operatorname{sign}[\operatorname{Im} \lambda]=\operatorname{sign}[\operatorname{Im} k]$, and we will define $\lambda(k)$ on $k \in\left[-q_{0}, q_{0}\right]$ so that it is continuous from above. Note $\lambda(k)$ is real-valued for $k \in \Sigma$, with $\Sigma=\mathbb{R} \backslash\left(-q_{0}, q_{0}\right)=$ $\left(-\infty,-q_{0}\right] \cup\left[q_{0}, \infty\right)$. We then define $\Phi_{-}(x, t, k)$ as the matrix solution of both parts of the Lax pair such that

$$
\Phi_{-}(x, t, k)=E_{-}(k) \mathrm{e}^{-i \theta_{-}(x, t, k) \sigma_{3}}+o(1), \quad x \rightarrow-\infty,
$$

where

$$
\theta_{-}(x, t, k)=\lambda x+2 k \lambda t
$$

Obviously $\Phi_{-}(x, t, k)$ is well-defined $\forall k \in \Sigma$. Moreover, using standard arguments one can show that its columns $\Phi_{-, 1}(x, t, k)$ and $\Phi_{-, 2}(x, t, k)$ admit analytic continuation to $\operatorname{Im} k>0$ and $\operatorname{Im} k<0$, respectively.

Importantly, one can also define $\Phi_{-, 1}(x, t, k)$ and $\Phi_{-, 2}(x, t, k)$ on the branch cut $k \in\left(-q_{0}, q_{0}\right)$ by taking the limit from the UHP and the LHP, respectively. As a result, all four column eigenfunctions [i.e., $\Phi_{ \pm, 1}(x, t, k)$ and $\left.\Phi_{ \pm, 2}(x, t, k)\right]$ are defined $\forall k \in \mathbb{R}$. Note however that, while the limit of $\Phi_{-, 1}(x, t, k)$ to $k \in\left(-q_{0}, q_{0}\right)$ from the UHP is straightforward (because it is continuous from above), the evaluation of the limit of $\Phi_{-, 2}(x, t, k)$ to $k \in\left(-q_{0}, q_{0}\right)$ from the LHP requires some care as a result of the discontinuity of $\lambda(k)$ when taking the limit to $\left(-q_{0}, q_{0}\right)$ from below. This has important consequences in the development of the IST, so we take a closer look at such limits.

Auxiliary eigenfunctions. Note that for $k \in\left(-q_{0}, q_{0}\right), \lim _{\epsilon \downarrow 0} \lambda(k \pm i \epsilon)= \pm \lambda(k)$. As in the asymmetric case, for $k \in \Sigma$ one could just as well choose the opposite sign for $\lambda(k)$. To make this statement more precise, we introduce a set of auxiliary eigenfunctions $\tilde{\Phi}_{-}(x, t, k)$ such that

$$
\tilde{\Phi}_{-}(x, t, k)=\tilde{E}_{-}(k) \mathrm{e}^{-i \tilde{\theta}_{-}(x, t, k)}+o(1), \quad x \rightarrow-\infty,
$$

where $\tilde{\theta}_{-}(x, t, k)=-\lambda(x+2 k t)=-\theta_{-}(x, t, k)$ and $\tilde{E}_{-}(k)=I-i /(k-\lambda) \sigma_{3} Q_{-}$. It should then be clear that: (i) The regions of analyticity for the columns of $\tilde{\Phi}_{-}(x, t, k)$ are the opposite of those of $\Phi_{-}(x, t, k)$. (ii) In particular, for $k \in\left[-q_{0}, q_{0}\right], \tilde{\Phi}_{-, 2}$ is continuous from above. (iii) Importantly,

$$
\lim _{\epsilon \uparrow 0} \Phi_{-, 2}(x, t, k+i \epsilon)=\tilde{\Phi}_{-, 2}(x, t, k), \quad k \in\left[-q_{0}, q_{0}\right] .
$$


Our next task is to express $\tilde{\Phi}_{-, 2}(x, t, k)$ in terms of known eigenfunctions. To this end, we note that, for all $\operatorname{Im} k \geqslant 0$, we have $\tilde{\Phi}_{-, 2}(x, t, k)=\left(-i q_{-} /(k-\lambda), 1\right)^{T} \mathrm{e}^{-i \theta_{-}(x, t, k)}+o(1)$ as $x \rightarrow-\infty$. On the other hand, $\Phi_{-, 1}(x, t, k)=$ $\left(1,-i r_{-} /(k+\lambda), 1\right)^{T} \mathrm{e}^{-i \theta_{-}(x, t, k)}+o(1)$ as $x \rightarrow-\infty$ for all $\operatorname{Im} k \geqslant 0$. Moreover, $\Phi_{-, 1}(x, t, k)$ and $\tilde{\Phi}_{-, 2}(x, t, k)$ are both solutions of the scattering problem, and they have the same exponential dependence; furthermore, $\operatorname{Im} \lambda(k)>0$ for all $k \in\left(-q_{0}, q_{0}\right)$ and therefore $\mathrm{e}^{-i \theta_{-}(x, t, k)} \rightarrow 0$ as $x \rightarrow-\infty$. Therefore, $\Phi_{-, 1}(x, t, k)$ and $\tilde{\Phi}_{-, 2}(x, t, k)$ must be proportional. Indeed, $\left(-i q_{-} /(k-\lambda), 1\right)=c\left(1,-i q_{-}^{*} /(k+\lambda), 1\right)$ for $c=-i q_{-} /(k-\lambda)$. Combining these results, we finally obtain:

$$
\Phi_{-, 2}(x, t, k)=\lim _{\epsilon \uparrow 0} \Phi_{-, 2}(x, t, k+i \epsilon)=-\frac{i q_{-}}{k-\lambda} \Phi_{-, 1}(x, t, k), \quad k \in\left(-q_{0}, q_{0}\right) .
$$

Scattering matrix. Recall that all of the Jost eigenfunctions $\Phi_{ \pm, 1}$ and $\Phi_{ \pm, 2}$ are defined $\forall k \in \mathbb{R}$. Since $\operatorname{tr} X=\operatorname{tr} T=$ 0 , the determinant of any matrix solution of the Lax pair is independent of $x$ and $t$. For the Jost eigenfunctions, this determinant is most easily evaluated in the limit $x \rightarrow \pm \infty$. In this way one obtains $\operatorname{det} \Phi_{+}(x, t, k)=1$ for all $k \in \mathbb{R}$. Also, for $k \in \Sigma$ we have $\operatorname{det} \Phi_{-}(x, t, k)=2 \lambda /(k+\lambda) \equiv 1 / d_{-}(k)$. On the other hand, for $k \in\left(-q_{0}, q_{0}\right)(\mathrm{C} .3)$ implies det $\Phi_{-}(x, t, k)=0$.

However, since $\Phi_{+}(x, t, k)$ is a fundamental matrix solution of the Lax pair $\forall k \in \mathbb{R}$, one can express the columns of $\Phi_{-}(x, t, k)$ as a linear combinations of those of $\Phi_{+}(x, t, k)$ :

$$
\Phi_{-}(x, t, k)=\Phi_{+}(x, t, k) S(k), \quad \forall k \in \mathbb{R}
$$

As in the symmetric case, the scattering matrix $S(k)$ thus defined is independent of time, owing to the fact that $\Phi_{ \pm}(x, t, k)$ are simultaneous solutions of the Lax pair. Moreover, since $\operatorname{det} \Phi_{+}(x, t, k)=1 \forall k \in \mathbb{R}$ we have $\operatorname{det} S(k)=\operatorname{det} \Phi_{-}(x, t, k)$ for all $k \in \mathbb{R}$. That is,

$$
\operatorname{det} S(k)= \begin{cases}1 / d_{-}(k), & k \in \Sigma, \\ 0, & k \in\left(-q_{0}, q_{0}\right) .\end{cases}
$$

In column form (C.3) is, for all $k \in \mathbb{R}$,

$$
\begin{aligned}
& \Phi_{-, 1}(x, t, k)=s_{11}(k) \Phi_{+, 1}(x, t, k)+s_{21}(k) \Phi_{+, 2}(x, t, k), \\
& \Phi_{-, 2}(x, t, k)=s_{12}(k) \Phi_{+, 1}(x, t, k)+s_{22}(k) \Phi_{+, 2}(x, t, k) .
\end{aligned}
$$

In turn, using (C.3e), one can also express the individual scattering coefficients as Wronskians. Explicitly, for all $k \in \mathbb{R}$,

$$
\begin{gathered}
s_{11}(k)=\operatorname{Wr}\left(\Phi_{-, 1}(x, t, k), \Phi_{+, 2}(x, t, k)\right), \quad s_{12}(k)=\operatorname{Wr}\left(\Phi_{-, 2}(x, t, k), \Phi_{+, 2}(x, t, k)\right), \\
s_{21}(k)=\operatorname{Wr}\left(\Phi_{+, 1}(x, t, k), \Phi_{-, 1}(x, t, k)\right), \quad s_{22}(k)=\operatorname{Wr}\left(\Phi_{+, 1}(x, t, k), \Phi_{-, 2}(x, t, k)\right) .
\end{gathered}
$$

For future convenience, we also introduce the reflection coefficients

$$
\rho_{1}(k)=s_{21}(k) / s_{11}(k), \quad \rho_{2}(k)=s_{12}(k) / s_{22}(k), \quad k \in \mathbb{R} .
$$

Finally, we note that unlike the case of symmetric NZBC, all Jost eigenfunctions and scattering coefficients remain finite at the branch points.

It is worthwhile to note that (C.3) is usually referred to as the scattering relation from the right, and, as in the case of ZBC and symmetric NZBC, one can equivalently define a scattering relation from the left as

$$
\Phi_{+}(x, t, k)=\Phi_{-}(x, t, k) R(k) .
$$

The corresponding reflection coefficients are $r_{1}(k)=s_{12}(k) / s_{22}(k)$ and $r_{2}(k)=s_{12}(k) / s_{11}(k)$. Unlike the case of ZBC and symmetric NZBC, however, the formulations from the left and from the right are not equivalent. In particular, while $\operatorname{det} \Phi_{+}(x, t, k)=1 \forall k \in \mathbb{R} \operatorname{det} \Phi_{-}(x, t, k)=0$ for all $k \in\left[-q_{0}, q_{0}\right]$. Thus (C.3) only holds for 
$k \in \Sigma$ (because it is only there that $\Phi_{-}(x, t, k)$ is a fundamental matrix solution of the Lax pair). Nonetheless, one can use the Wronskian relations

$$
\begin{array}{ll}
r_{11}(k)=d_{-}(k) \operatorname{Wr}\left(\Phi_{+, 1}(x, t, k), \Phi_{-, 2}(x, t, k)\right), & r_{12}(k)=d_{-}(k) \operatorname{Wr}\left(\Phi_{+, 2}(x, t, k), \Phi_{-, 2}\right)(x, t, k), \\
r_{21}(k)=d_{-}(k) \operatorname{Wr}\left(\Phi_{-, 1}(x, t, k), \Phi_{+, 1}(x, t, k)\right), & r_{22}(k)=d_{-}(k) \operatorname{Wr}\left(\Phi_{-, 1}(x, t, k), \Phi_{+, 2}(x, t, k)\right),
\end{array}
$$

which hold for $k \in \Sigma$, as a a definition of all scattering coefficients $r_{i j}$ for all $k \in\left(-q_{0}, q_{0}\right)$. Of course, for the analytic scattering coefficients $r_{11}(k)$ and $r_{22}(k)$ this definition coincides with the value obtained by their analytic continuation. Note however that, even though all the pieces (C.3) are also defined for $k \in\left(-q_{0}, q_{0}\right)$, the scattering relation is not valid there. Obviously, $R(k)=S^{-1}(k)$ for all $k \in \Sigma$. Entrywise, one has:

$$
r_{11}(k)=d_{-}(k) s_{22}(k), r_{12}(k)=-d_{-}(k) s_{12}(k), r_{21}(k)=-d_{-}(k) s_{21}(k), \quad r_{22}(k)=d_{-}(k) s_{11}(k), \quad k \in \Sigma .
$$

As usual, these relations can be extended to the UHP/LHP for the analytic scattering coefficients.

Symmetries. As before, the scattering problem admits a symmetry under the map $k \mapsto k^{*}$. The symmetry of $\Phi_{ \pm}(x, t, k)$ is the same as in the case of ZBC:

$$
\Phi_{+}(x, t, k)=\sigma_{1} \Phi_{+}^{*}\left(x, t, k^{*}\right) \sigma_{1} .
$$

In matrix form, (C.3) holds only where all columns are defined, i.e., $k \in \mathbb{R}$. However, its first and second columns can be extended to the UHP and LHP, respectively. A similar symmetry holds for $\Phi_{-}(x, t, k)$ :

$$
\Phi_{-}(x, t, k)=\sigma_{1} \Phi_{-}^{*}\left(x, t, k^{*}\right) \sigma_{1} .
$$

Here, however, the situation is complicated by the fact that $\Phi_{-}(x, t, k)$ is only defined as a matrix for $k \in \Sigma$, and, more importantly, that $\lambda(k)$ is discontinuous across $k \in\left(-q_{0}, q_{0}\right)$. In other words, the relation $\lambda\left(k^{*}\right)=\lambda^{*}(k)$ [which is needed to derive (C.3)] only holds for $k \in \mathbb{C} \backslash\left(-q_{0}, q_{0}\right)$. Thus, (C.3) does not hold for $k \in\left(-q_{0}, q_{0}\right)$. Indeed, we have already seen that for $k \in\left(-q_{0}, q_{0}\right)$ the correct symmetry is (C.3).

The corresponding symmetries of the scattering coefficients can be obtained from (C.3):

$$
S(k)=\sigma_{1} S^{*}\left(k^{*}\right) \sigma_{1} .
$$

Of course, as a matrix equation (C.3) only holds for $k \in \Sigma$, where both (C.3) and (C.3) apply. In particular,

$$
s_{12}(k)=s_{21}^{*}\left(k^{*}\right), \quad k \in \Sigma .
$$

However, one can use the Schwartz reflection symmetry to extend the diagonal entries:

$$
s_{11}(k)=s_{22}^{*}\left(k^{*}\right), \quad k \in \Sigma \cup \mathbb{C}^{+} \text {. }
$$

On the other hand, for $k \in\left(-q_{0}, q_{0}\right)$ one must replace (C.3) with (C.3). Then, comparing the first and the second of (C.3e) one obtains

$$
\left(s_{12}(k), s_{22}(k)\right)=-\frac{i q_{-}}{k-\lambda}\left(s_{11}(k), s_{21}(k)\right), \quad k \in\left(-q_{0}, q_{0}\right) .
$$

Recalling the definition (C.3) of the reflection coefficients one then obtains

$$
\begin{gathered}
\rho_{1}(k)=\rho_{2}^{*}(k), \quad k \in \Sigma, \\
\rho_{1}(k) \rho_{2}(k)=1, \quad k \in\left(-q_{0}, q_{0}\right) .
\end{gathered}
$$


Asymptotic behavior as $k \rightarrow \infty$. First of all, note $\lambda(k)=k-q_{0}^{2} /(2 k)+O\left(1 / k^{2}\right)$ as $k \rightarrow \infty$. Let us remove the exponential oscillations by introducing as usual modified eigenfunctions as

$$
\mu_{ \pm}(x, t, k)=\Phi_{ \pm}(x, t, k) \mathrm{e}^{i \theta_{ \pm}(x, t, k) \sigma_{3}} .
$$

The asymptotics of $\Phi_{+}(x, t, k)$ are of course identical to the case of ZBC. Similarly, the asymptotics of $\Phi_{-}(x, t, k)$ is the same as that of the case of symmetric NZBC. Overall, we have:

$$
\mu_{ \pm}(x, t, k)=I \mp \frac{1}{2 i k} \sigma_{3} Q(x, t)+\text { h.o.t. }, \quad k \rightarrow \infty,
$$

where "h.o.t." denotes $O(1 / k)$ diagonal terms and $O\left(1 / k^{2}\right)$ off-diagonal terms. In particular,

$$
q(x, t)=-2 i \lim _{k \rightarrow \infty} k \mu_{+, 12}(x, t, k) .
$$

This relation will provide the key for reconstructing the potential from the solution of the RHP. Also, from the Wronskian definitions (C.3f) of the scattering coefficients, it follows

$$
s_{11}(k)=1+O(1 / k), \quad s_{21}(k)=\frac{i q_{-}^{*}}{2 k}+O\left(1 / k^{2}\right) \quad k \rightarrow \infty .
$$

Discrete spectrum. It is straightforward to show that, as in the case of symmetric NZBC, no zeros of $s_{11}(k)$ can occur in the continuous spectrum $k \in \Sigma$. Moreover, similar arguments as in the fully asymmetric case, show that no zeros of $s_{11}(k)$ and $s_{12}(k)$ can lie in the "DSW" spectrum $k \in\left(-q_{0}, q_{0}\right)$. As a result, we obtain that no discrete spectrum can be present in the problem with one-sided NZBC.

Step IC. We now consider the IC (C.2). In this case we can solve the scattering problem exactly at $t=0$. And since the scattering matrix is independent of time, this is all is needed for the IVP.

The scattering problem at time $t=0$ has the form: $v_{x}=X_{ \pm} v$ for $x \gtrless 0$, with piecewise constant coefficients

$$
X_{ \pm}=-i k \sigma_{3}+Q_{ \pm}, \quad Q_{-}=\left(\begin{array}{cc}
0 & q_{-} \\
q_{-}^{*} & 0
\end{array}\right), \quad Q_{+}=0 .
$$

One can easily find exact solutions which yield explicit, simple representations for the Jost solutions at time $t=0$ over half of the real $x$-axis:

$$
\begin{gathered}
\Phi_{-}(x, 0, k)=E_{-}(k) \mathrm{e}^{-i \theta_{-}(x, t, k) \sigma_{3}} \quad x<0, \\
\Phi_{+}(x, 0, k)=\mathrm{e}^{-i \theta_{+}(x, t, k) \sigma_{3}} \quad x>0,
\end{gathered}
$$

with $\theta_{ \pm}(x, t, k)$ and $E_{-}(k)$ as before. The form of the Jost solution beyond these domains can then be obtained by writing each Jost solution as an appropriate linear combination of the fundamental matrix solution of the scattering problem in each region and then imposing continuity at the boundary. On the other hand, for the purpose of determining the scattering data, we can simply evaluate (C.3) at $(x, t)=(0,0)$, obtaining

$$
S(k)= \begin{cases}E_{-}(k), & k \in \Sigma \\ E_{0}(k), & k \in\left(-q_{0}, q_{0}\right) .\end{cases}
$$

where the matrix

$$
E_{o}(k)=E_{-, 1}(k)\left(1,-i q_{-} /(k-\lambda)\right)=\left(\begin{array}{cc}
1 & -i q_{-} /(k-\lambda) \\
i q_{-}^{*} /(k+\lambda) & 1
\end{array}\right)
$$

was obtained using (C.3). The reflection coefficients are then

$$
\begin{aligned}
& \rho_{1}(k)=i q_{-}^{*} /(k+\lambda), \quad k \in \mathbb{R}, \\
& \rho_{2}(k)= \begin{cases}-i q_{-} /(k+\lambda), & k \in \Sigma, \\
-i q_{-} /(k-\lambda), & k \in\left(-q_{0}, q_{0}\right) .\end{cases}
\end{aligned}
$$

Once can verify that, since $s_{11}(k)=s_{22}(k)=1$, there are no discrete eigenvalues. 


\section{C.2. Inverse problem}

We introduce a sectionally meromorphic matrix similarly to (B.7) in the case of symmetric NZBC, namely:

$$
M(x, t, k)=\left\{\begin{array}{l}
\left(\frac{\mu_{-, 1}}{s_{11}}, \mu_{+, 2}\right)=\left(\frac{\Phi_{-, 1}}{s_{11}}, \Phi_{+, 2}\right) \mathrm{e}^{i \Theta}, \quad \operatorname{Im} k>0, \\
\left(\mu_{+, 1}, \frac{\mu_{-, 2}}{s_{22}}\right)=\left(\Phi_{+, 1}, \frac{\Phi_{-, 2}}{s_{22}}\right) \mathrm{e}^{i \tilde{\Theta}}, \quad \operatorname{Im} k<0,
\end{array}\right.
$$

where

$$
\Theta(x, t, k)=\operatorname{diag}\left(\theta_{-}(x, t, k),-\theta_{+}(x, t, k)\right), \quad \tilde{\Theta}(x, t, k)=\operatorname{diag}\left(\theta_{+}(x, t, k),-\theta_{-}(x, t, k)\right),
$$

with $\theta_{ \pm}(x, t, k)$ given by $(\mathrm{C} .3 \mathrm{db})$ and (C.3b). It is easy to verify from the asymptotic of the eigenfunctions that a canonical normalization condition at infinity holds for $M(x, t, k)$, i.e.,

$$
M(x, t, k)=I+O(1 / k) \quad k \rightarrow \infty .
$$

The goal is then to determine a jump condition similar to (B.8), where $M^{ \pm}(x, t, k)$ are the limiting values of $M(x, t, k)$ as $\operatorname{Im} k \rightarrow 0^{ \pm}$. The starting point is, as always, the scattering relation (C.3). Separating the eigenfunctions analytic in the UHP from those analytic in the LHP one obtains

$$
\left(\frac{\Phi_{-, 1}(x, t, k)}{s_{11}(k)}, \Phi_{+, 2}(x, t, k)\right)=\left(\Phi_{+, 1}(x, t, k), \frac{\Phi_{-, 2}(x, t, k)}{s_{22}(k)}\right) \tilde{V}(k), \quad k \in \mathbb{R},
$$

where the "core" jump matrix $\tilde{V}(k)$ is

$$
\tilde{V}(k)=\left(\begin{array}{cc}
1-\rho_{1}(k) \rho_{2}(k) & -\rho_{2}(k) \\
\rho_{1}(k) & 1
\end{array}\right), \quad k \in \mathbb{R} .
$$

Note that the expression for $\tilde{V}(k)$ is the formally the same for $k \in \Sigma$ and $k \in\left(-q_{0}, q_{0}\right)$. The difference is in the symmetries of the scattering coefficients. Indeed, recalling (C.3g) and letting $\rho(k)=\rho_{1}(k)$ for all $k \in \mathbb{R}$ we have

$$
\tilde{V}(k)=\left\{\begin{array}{ll}
\left(\begin{array}{cc}
1-|\rho(k)|^{2} & -\rho^{*}(k) \\
\rho(k) & 1
\end{array}\right), & k \in \Sigma, \\
0 & -1 / \rho(k) \\
\rho(k) & 1
\end{array}\right), \quad k \in\left(-q_{0}, q_{0}\right) .
$$

The jump condition is then given by $M^{+}(x, t, k)=M^{-}(x, t, k) V(x, t, k)$, where the full jump matrix $V(x, t, k)$ is obtained from $\tilde{V}(k)$ as

$$
V(x, t, k)=\left(\lim _{\epsilon \uparrow 0} \mathrm{e}^{i \Theta \tilde{\Theta}(x, t, k+i \epsilon)}\right)^{-1} \tilde{V}(k) \lim _{\epsilon \downarrow 0} \mathrm{e}^{i \Theta(x, t, k+i \epsilon)} .
$$

Explicitly, recalling (C.12) and the discontinuous behavior of $\lambda(k)$ on $\left(-q_{0}, q_{0}\right)$ from below, we have

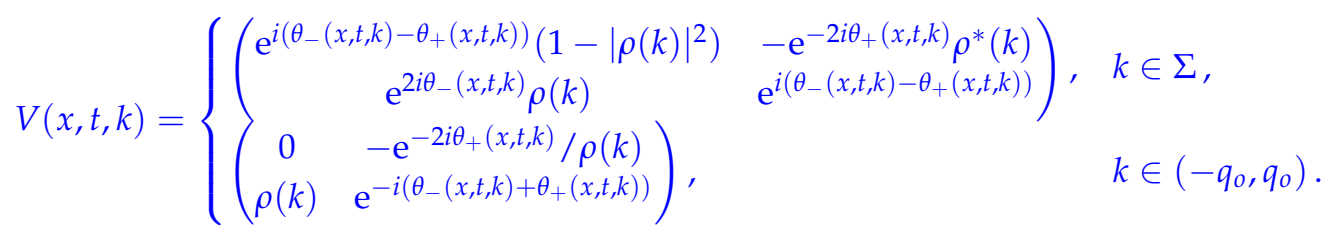

Importantly, the full jump matrix is not unimodular, unlike the case of ZBC or symmetric NZBC. Explicitly,

$$
\operatorname{det} V(x, t, k)= \begin{cases}\mathrm{e}^{2 i\left(\theta_{-}(x, t, k)-\theta_{+}(x, t, k)\right),} & k \in \Sigma, \\ \mathrm{e}^{-2 i \theta_{+}(x, t, k),} & k \in\left(-q_{0}, q_{0}\right) .\end{cases}
$$

However, det $V(x, t, k) \rightarrow 1$ as $k \rightarrow \infty$ - as it must in order to be consistent with the normalization (C.2) in both the UHP and LHP. The RHP can now be formally solved by subtracting the asymptotic behavior at infinity applying Cauchy projectors. We omit the details since they are similar to the cases previously presented. 


\section{C.3. Conversion between RHP from the left and from the right}

Recall that one can equivalently write the scattering relation as $\Phi_{-}(x, t, k)=\Phi_{+}(x, t, k) S(k)$, like we have done here, or as $\Phi_{+}(x, t, k)=\Phi_{-}(x, t, k) R(k)$. Correspondingly, one can formulate two different RHPs: one "from the right", which is the one we used here, and one "from the right". The choice breaks the left/right symmetry of the NLS equation. On the other hand, it is possible to convert from one RHP to the other, as we discuss next. The issue is relevant in our case because, unlike the case of ZBC and symmetric NZBC, with asymmetric NZBC the Jost eigenfunctions from the left and from the right possess different domains of analyticity. This in turn determines where it is possible to define the reflection coefficients appearing in the jump condition for the RHP.

Recall that the sectionally meromorphic matrix $M^{ \pm}(x, t, k)$ for the RHP in section 4 was defined in (C.2). On the other hand, in the RHP from the left is constructed using the sectionally meromorphic matrix

$$
\check{M}(x, t, k)= \begin{cases}\left(\mu_{-, 1}(x, t, k), \frac{\mu_{+, 2}(x, t, k)}{r_{22}(k)}\right), & \operatorname{Im} k>0, \\ \left(\frac{\mu_{+, 1}(x, t, k)}{r_{11}(k)}, \mu_{-, 2}(x, t, k)\right), & \operatorname{Im} k<0 .\end{cases}
$$

We have $M(x, t, k)=\check{M}(x, t, k) D(k)$, where

$$
D(k)= \begin{cases}\operatorname{diag}\left(1 / s_{11}(k), r_{22}(k)\right), & \operatorname{Im} k>0, \\ \operatorname{diag}\left(r_{11}(k), 1 / s_{22}(k)\right), & \operatorname{Im} k<0 .\end{cases}
$$

Correspondingly, $\check{M}(x, t, k)$ satisfies the jump condition

$$
\check{M}^{+}(x, t, k)=\check{M}^{-}(x, t, k) \breve{V}(x, t, k), \quad k \in \mathbb{R},
$$

where

$$
\check{V}(x, t, k)=D^{-}(k) V(x, t, k)\left(V^{+}(x, t, k)\right)^{-1}, \quad k \in \mathbb{R} .
$$

Recalling the relations (C.3) between the entries of $S(k)$ and $R(k)$, straightforward algebra yields $\breve{V}(0,0, k)$. The matrix $\breve{V}(x, t, k)$ is then obtained from $V(0,0, k)$ by conjugating with the appropriate matrix exponentials.

\section{Appendix D. Asymptotic behavior of eigenfunctions and scattering data as $k \rightarrow \infty$}

As usual, in order to normalize the RHP one must evaluate the asymptotic behavior of the eigenfunctions and scattering data as $k \rightarrow \infty$. Below we show that, if $q(x, t)$ is differentiable with respect to $x$ and $q_{x}(\cdot, t) \in L^{1}(\mathbb{R})$, the analytic columns of the modified Jost eigenfunfunctions exhibit the following asymptotic behavior as $k \rightarrow \infty$ in each half plane:

$$
\begin{array}{ll}
\mu_{-, 11}(x, t, k)=1+O(1 / k), \quad & \mu_{-, 21}(x, t, k)=\frac{i q^{*}(x, t)}{2 k}+O\left(1 / k^{2}\right), \quad k \rightarrow \infty, \operatorname{Im} k \geqslant 0, \\
\mu_{-, 11}(x, t, k)=\frac{q(x, t)}{q_{-}(t)}+O(1 / k), & \mu_{-, 21}(x, t, k)=\frac{2 i k}{q_{-}(t)}+O(1), \quad k \rightarrow \infty, \operatorname{Im} k<0 .
\end{array}
$$

Moreover,

$$
\begin{gathered}
\mu_{+, 12}(x, t, k)=-\frac{i q(x, t)}{2 k}+O\left(1 / k^{2}\right), \quad \mu_{+, 22}(x, t, k)=1+O(1 / k), \quad k \rightarrow \infty, \operatorname{Im} k \geqslant 0, \\
\mu_{+, 12}(x, t, k)=-\frac{2 i k}{q_{+}^{*}(t)}+O(1), \quad \mu_{+, 22}(x, t, k)=\frac{q^{*}(x, t)}{q_{+}^{*}(t)}+O(1 / k), \quad k \rightarrow \infty, \operatorname{Im} k<0 .
\end{gathered}
$$


We present the calculations for $\mu_{-, 1}(x, t, k)$ in detail; those for $\mu_{+, 2}(x, t, k)$ are similar. We already know that the first column of $\mu_{-}(x, t, k)$ admits a uniformly convergent Neumann series representation in the UHP for its integral equation, which we rewrite as:

$$
\mu_{-, 1}(x, t, k)=\left(\begin{array}{c}
1 \\
\frac{i q_{-}^{*}(t)}{k+\lambda_{-}(k)}
\end{array}\right)+\int_{-\infty}^{x} G(x-y, t, k) \Delta Q_{-}(y, t) \mu_{-, 1}(y, t, k) \mathrm{d} y,
$$

where

$$
G(\xi, t, k)=E_{-}(k, t)\left(\begin{array}{cc}
1 & 0 \\
0 & \mathrm{e}^{2 i \lambda_{-}(k) \xi}
\end{array}\right) E_{-}^{-1}(k, t)=\frac{1}{2}\left(1+\mathrm{e}^{2 i \lambda_{-}(k) \xi}\right) I+\frac{1}{2 \lambda_{-}(k)}\left(1-\mathrm{e}^{2 i \lambda_{-}(k) \xi}\right)\left[k \sigma_{3}+Q_{-}(t)\right]
$$

It is also convenient to separate (D.3) into its components, obtaining:

$$
\begin{gathered}
\mu_{-, 11}(x, t, k)=1 \\
+\frac{i q_{-}(t)}{2 \lambda_{-}(k)} \int_{-\infty}^{x} \Delta q_{-}^{*}(y, t) \mu_{-, 11}(y, t, k) \mathrm{d} y-\frac{i q_{-}(t)}{2 \lambda_{-}(k)} \int_{-\infty}^{x} \mathrm{e}^{2 i \lambda_{-}(k)(x-y)} \Delta q_{-}^{*}(y, t) \mu_{-, 11}(y, t, k) \mathrm{d} y \\
+\frac{k+\lambda_{-}(k)}{2 \lambda_{-}(k)} \int_{-\infty}^{x} \Delta q_{-}(y, t) \mu_{-, 21}(y, t, k) \mathrm{d} y-\frac{k-\lambda_{-}(k)}{2 \lambda_{-}(k)} \int_{-\infty}^{x} \mathrm{e}^{2 i \lambda_{-}(k)(x-y)} \Delta q_{-}(y, t) \mu_{-, 21}(y, t, k) \mathrm{d} y, \\
\mu_{-, 21}(x, t, k)=\frac{i q_{-}^{*}(t)}{k+\lambda_{-}(k)} \\
+\frac{i q_{-}^{*}(t)}{2 \lambda_{-}(k)} \int_{-\infty}^{x} \Delta q_{-}(y, t) \mu_{-, 21}(y, t, k) \mathrm{d} y-\frac{i q_{-}^{*}(t)}{2 \lambda_{-}(k)} \int_{-\infty}^{x} \mathrm{e}^{2 i \lambda_{-}(k)(x-y)} \Delta q_{-}(y, t) \mu_{-, 21}(y, t, k) \mathrm{d} y \\
-\frac{k-\lambda_{-}(k)}{2 \lambda_{-}(k)} \int_{-\infty}^{x} \Delta q_{-}^{*}(y, t) \mu_{-, 11}(y, t, k) \mathrm{d} y+\frac{k+\lambda_{-}(k)}{2 \lambda_{-}(k)} \int_{-\infty}^{x} \mathrm{e}^{2 i \lambda_{-}(k)(x-y)} \Delta q_{-}^{*}(y, t) \mu_{-, 11}(y, t, k) \mathrm{d} y,
\end{gathered}
$$

By Riemann-Lebesgue, the second integral in the right-hand side (RHS) of (D.5a) always vanishes faster than the first one as $k \rightarrow \infty$, and similarly for the third and fourth integrals. The same argument applies to the first two integrals in the RHS of (D.5b), but not to the last two [since $k-\lambda_{-}(k)=O(1 / k)$ as $k \rightarrow \infty$ in the UHP]. Also, the first integral in the RHS of (D.5a) always vanishes faster than the left-hand side (LHS) of (D.5a) as $k \rightarrow \infty$, and the same holds for the first integral in the RHS of (D.5a) compared to its LHS. Substituting (D.5b) in the RHS of (D.5a) and (D.5a) in the RHS of (D.5b) we then obtain $\mu_{-, 11}(x, t, k)=1+O(1 / k)+O\left(\mu_{-, 21}(x, t, k) / k\right)$ and $\mu_{-, 21}(x, t, k)=O(1 / k)+O\left(\mu_{-, 11}(x, t, k) / k\right)$ as $k \rightarrow \infty$ in the UHP, implying $\mu_{-, 11}(x, t, k)=1+O(1 / k)$ and $\mu_{-, 21}(x, t, k)=O(1 / k)$, as expected.

The first of (D.1) is thus proved. We now note that, if the distributional derivative $\partial_{x} q(x, t)$ is in $L^{1}(\mathbb{R})$, integration by parts and the Riemann-Lebesgue lemma yield

$$
\int_{-\infty}^{x} \mathrm{e}^{2 i \lambda_{-}(k)(x-y)}\left(q(y, t)-q_{-}(t)\right) \mathrm{d} y=\frac{1}{2 i \lambda_{-}(k)}\left(q(x, t)-q_{-}(t)\right)+o(1 / k), \quad k \rightarrow \infty .
$$

Replacing (D.5a) in the last integral of (D.5b) and using (Appendix D) then finally yields the second of (D.1).

The calculations of the asymptotic behavior as $k \rightarrow \infty$ in the LHP are slightly more complicated by the fact that $k+\lambda=O(1 / k)$ in that limit [since $\lambda(k)=-k+O(1 / k)$ as $k \rightarrow \infty$ in the LHP]. Nonetheless, one can follow a similar logic to obtain (D.1b) and (D.2b)

Asymptotic expansions up to $O\left(1 / k^{n}\right)$ can also be iteratively obtained under the assumption that $\partial_{x}^{j} q(x, t) \in$ $L^{1}(\mathbb{R})$ for $j=1, \ldots, n$. It should also be noted that $\partial_{x} q(x, t) \in L^{1}(\mathbb{R})$ implies that $q(x, t)$ is absolutely continuous as a function of $x$, which is consistent with the second of (D.1). 
The asymptotic behavior of the modified eigenfunctions can now be used to derive the asymptotic behavior of $s_{11}(k, t)$ via (2.14). Explicitly:

$$
\begin{gathered}
s_{11}(k, t)=1+O(1 / k), \quad k \rightarrow \infty \wedge \operatorname{Im} k>0, \\
s_{11}(k, t)=\frac{q_{+}(t)}{q_{-}(t)}+O(1 / k), \quad k \rightarrow \infty \wedge \operatorname{Im} k<0 .
\end{gathered}
$$

Combining these expressions with (D.1) and (D.2) we obtain the asymptotic behavior of the meromorphic matrix in the RHP, namely (4.17).

In the case of symmetric NZBC, the calculations are similar, with a couple of caveats. The first is that in the symmetric case a phase rotation was applied to the the NLS equation to simplify the boundary conditions. This affects the time evolution of the Jost eigenfunctions. Also, in the symmetric case the Jost eigenfunctions were defined to be simultaneous solutions of both parts of the Lax pair, which makes all scattering coefficients independent of time. Finally, in the symmetric case we have $\lambda_{ \pm}=\lambda$. On the other hand, the asymptotic behavior of $M(x, t, k)$ as $k \rightarrow \infty$ turns out to be still given by (4.17), with $\lambda_{+}(k)$ replaced by $\lambda$ and $Q_{+}(t)$ replaced by $Q_{+}$.

\section{Appendix E. Proof that the discrete eigenvalues are simple}

Here we provide an explicit proof of the fact that, even in the case of asymmetric NZBC, $s_{11}(k)$ can have only simple zeros. Recall that the prime denotes differentiation with respect to $k$. Using (2.14) we have that

$$
s_{11}^{\prime}\left(k_{j}, t\right)=d_{+}\left(k_{j}\right)\left[\operatorname{Wr}\left(\Phi_{-, 1}^{\prime}, \Phi_{+, 2}\right)\left(x, t, k_{j}\right)+\operatorname{Wr}\left(\Phi_{-, 1}, \Phi_{+, 2}^{\prime}\right)\left(x, t, k_{j}\right)\right] .
$$

Since $\Phi_{ \pm}$solve the scattering ODE, $\left(\Phi_{ \pm}\right)_{x}^{\prime}=-i \sigma_{3} \Phi_{ \pm}+X \Phi_{ \pm}$, and:

$$
\partial_{x} \operatorname{Wr}\left(\Phi_{-, 1}^{\prime}, \Phi_{+, 2}\right)(x, t, k)=-i \operatorname{Wr}\left(\sigma_{3} \Phi_{-, 1}, \Phi_{+, 2}\right)+\operatorname{Wr}\left(X \Phi_{-, 1}^{\prime}, \Phi_{+, 2}\right)+\operatorname{Wr}\left(\Phi_{-, 1}^{\prime}, X \Phi_{+, 2}\right) .
$$

We use that $-X=X^{-1} \operatorname{det} X$, so $\operatorname{Wr}(X u, v)=-\operatorname{Wr}(u, X v)$. Thus (E.1) reduces to

$$
\partial_{x} \operatorname{Wr}\left(\Phi_{-, 1}^{\prime}, \Phi_{+, 2}\right)=-i \operatorname{Wr}\left(\sigma_{3} \Phi_{-, 1}, \Phi_{+, 2}\right),
$$

and similarly

$$
\partial_{x} \operatorname{Wr}\left(\Phi_{-, 1}, \Phi_{+, 2}^{\prime}\right)=i \operatorname{Wr}\left(\sigma_{3} \Phi_{+, 2}, \Phi_{-, 1}\right) .
$$

Evaluating at $k=k_{j}$, and recalling (2.40) yields:

$$
\begin{gathered}
\partial_{x} \operatorname{Wr}\left(\Phi_{-, 1}^{\prime}, \Phi_{+, 2}\right)\left(x, t, k_{j}\right)=-i b_{j}(t) \operatorname{Wr}\left(\sigma_{3} \Phi_{+, 2}, \Phi_{+, 2}\right)\left(x, t, k_{j}\right) \\
\partial_{x} \operatorname{Wr}\left(\Phi_{-, 1}, \Phi_{+, 2}^{\prime}\right)\left(x, t, k_{j}\right)=i b_{j}(t) \operatorname{Wr}\left(\sigma_{3} \Phi_{+, 2}, \Phi_{+, 2}\right)\left(x, t, k_{j}\right)
\end{gathered}
$$

Using the symmetry (2.21b) we have $\operatorname{Wr}\left(\sigma_{3} \Phi_{+, 2}, \Phi_{+, 2}\right)\left(x, t, k_{j}\right)=-i q_{+}(t)\left\|\Phi_{+, 2}\left(x, t, k_{j}\right)\right\|^{2} /\left[k_{j}+\lambda_{+, j}\right]$, where $\left\|\Phi_{+, 2}\left(x, t, k_{j}\right)\right\|^{2}=\left|\Phi_{+, 12}\left(x, t, k_{j}\right)\right|^{2}+\left|\Phi_{+, 22}\left(x, t, k_{j}\right)\right|^{2}$. Thus

$$
\begin{aligned}
\operatorname{Wr}\left(\Phi_{-, 1}^{\prime}, \Phi_{+, 2}\right)\left(x, t, k_{j}\right) & =\frac{i b_{j}(t) q_{+}(t)}{k_{j}+\lambda_{+, j}} \int_{x}^{\infty}\left\|\Phi_{+, 2}\left(y, t, k_{j}\right)\right\|^{2} \mathrm{~d} y, \\
\operatorname{Wr}\left(\Phi_{-, 1}, \Phi_{+, 2}^{\prime}\right)\left(x, t, k_{j}\right) & =\frac{i b_{j}(t) q_{+}(t)}{k_{j}+\lambda_{+, j}} \int_{-\infty}^{x}\left\|\Phi_{+, 2}\left(y, t, k_{j}\right)\right\|^{2} \mathrm{~d} y .
\end{aligned}
$$

The above equations show that $\partial_{k} \operatorname{Wr}\left(\Phi_{-, 1}, \Phi_{+, 2}\right)\left(x, t, k_{j}\right)$ is non-zero, implying that $s_{11}^{\prime}\left(k_{j}, t\right)$ is non-zero. 


\section{References}

[1] M. J. Ablowitz, D. J. Kaup, A. C. Newell, H. Segur, The inverse scattering transform - Fourier analysis for nonlinear problems, Stud. Appl. Math. 53 (1974) 249-315.

[2] S. P. Novikov, S. V. Manakov, L. P. Pitaevskii, V. E. Zakharov, Theory of solitons: The inverse scattering method, Plenum, New York, 1984.

[3] V. E. Zakharov, A. B. Shabat, Exact theory of two-dimensional self-focusing and one-dimensional self-modulation of waves in nonlinear media, Sov. Phys. JETP 34 (1972) 62-69.

[4] V. E. Zakharov, A. B. Shabat, Interaction between solitons in a stable medium, Sov. Phys. JETP 37 (1973) $823-828$.

[5] M. J. Ablowitz, H. Segur, Solitons and the inverse scattering transform, SIAM Studies in Applied Mathematics, Philadelphia, 1981.

[6] G. Biondini, B. Prinari, On the spectrum of the Dirac operator and the existence of discrete eigenvalues for the defocusing nonlinear Schrödinger equation, Stud. Appl. Math. 132 (2014) 138-159.

[7] F. Demontis, B. Prinari, C. van der Mee, F. Vitale, The inverse scattering transform for the defocusing nonlinear Schrödinger equation with nonzero boundary conditions, Stud. Appl. Math. 131 (2012) 1-40.

[8] L. D. Faddeev, L. A. Takhtajan, Hamiltonian methods in the theory of solitons, Springer, Berlin, 1987.

[9] G. Biondini, D. K. Kraus, Inverse scattering transform for the defocusing Manakov system with nonzero boundary conditions, SIAM J. Math. Anal. 47 (2015) 706-757.

[10] E. A. Kuznetsov, Solitons in a parametrically unstable plasma, Sov. Phys. Dokl. 22 (1977) 507.

[11] Y.-C. Ma, The perturbed plane-wave solutions of the cubic Schrödinger equation, Stud. Appl. Math. 60 (1979) $43-58$.

[12] M. Boiti, F. Pempinelli, The spectral transform for the NLS equation with left-right asymmetric boundary conditions, Nuovo Cimento A 69 (1982) 213-227.

[13] R. Jenkins, Regularization of a sharp shock by the defocusing nonlinear Schrödinger equation, Nonlinearity 28 (2015) 2131-2180.

[14] J. C. DiFranco, K. T.-R. McLaughlin, A nonlinear Gibbs-type phenomenon for the defocusing nonlinear Schrödinger equation, Oxford Univ. Press 8 (2005) 403-459.

[15] R. Buckingham, S. Venakides, Long-time asymptotics of the nonlinear Schrödinger equation shock problem, Commun. Pure Appl. Math. 60 (2007) 1349-1414.

[16] A. Boutet de Monvel, V. P. Kotlyarov, D. Shepelsky, Focusing NLS equation: long-time dynamics of step-like initial data, Int. Math. Res. Not. 7 (2011) 1613-1653.

[17] S. Kamvissis, D. Shepelsky, L. Zielinski, Robin boundary condition and shock problem for the focusing nonlinear Schrödinger equation, J. Nonlin. Math. Phys., 22, (2015), pp. 448-473.

[18] B. Prinari, M. J. Ablowitz, G. Biondini, Inverse scattering transform for the vector nonlinear Schrödinger equation with nonvanishing boundary conditions, J. Math. Phys. 47 (2006) 1-33.

[19] H. Segur, M. J. Ablowitz, Asymptotic solutions and conservation laws for the nonlinear Schrödinger equation. Part I, J. Math. Phys. 17 (1976) $710-713$.

[20] V. E. Zakharov, S. V. Manakov, Asymptotic behavior of nonlinear waves systems integrated by the inverse scattering method, Sov. Phys. JETP 44 (1976) 106-112.

[21] P. Deift, X. Zhou, A steepest descent method for oscillatory Riemann-Hilbert problems: Asymptotics for the mKdV equation, Ann. Math. 137 (1993) 295-368.

[22] X. Zhou, The Riemann-Hilbert problem and inverse scattering, SIAM J. Math. Anal. 20 (1989) 966-986.

[23] S. Kamvissis, Long time behavior for the focusing nonlinear Schroedinger equation, Commun. Math. Phys. 180 (1996) 325-341.

[24] A. R. Its, A. F. Ustinov, Temporal asymptotic solution of the Cauchy problem for the nonlinear Schrödinger equation with boundary conditions of the finite-density type, Sov. Phys. Dokl. 31 (1986) 893-895.

[25] A. H. Vartanian, Large-time continuum asymptotics of dark solitons, Inv. Prob. 16 (2000) L39-L46.

[26] A. H. Vartanian, Large-time asymptotics of solutions to the Cauchy problem for the defocusing nonlinear Schrödinger equation with finite density initial data; II Dark solitons on continua, Math. Phys. Anal. Geom. 5 (2002) 319-413.

[27] G. Biondini, G. Kovačič, Inverse scattering transform for the focusing nonlinear Schrödinger equation with nonzero boundary conditions, J. Math. Phys. 55 (2014) 031506.

[28] F. Demontis, B. Prinari, C. van der Mee, F. Vitale, The inverse scattering transform for the focusing nonlinear Schrödinger equation with nonzero boundary conditions, J. Math. Phys. 101505 (2014) 1-40.

[29] J. Satsuma, N. Yajima, Initial value problems of one-dimensional self-modulation of nonlinear waves in dispersive media, Progr. Theor. Phys. Suppl. 55 (1974) 284-306.

[30] G. Biondini, E. Fagerstrom, The integrable nature of modulational instability, SIAM J. Appl Math. 75 (2015) $136-163$.

[31] S. V. Manakov, On the theory of two-dimensional stationary self-focusing of electromagnetic waves, Zh. Eksp. Teor. Fiz. 65 (1973) 505-516.

[32] M. J. Ablowitz, B. Prinari, A. D. Trubatch, Discrete and continuous nonlinear Schrödinger systems, no. 302 in London Mathematical Society Lecture Note Series, Cambridge University Press, 2004.

[33] D. K. Kraus, G. Biondini, G. Kovačič, The focusing Manakov system with nonzero boundary conditions, Nonlinearity 28 (2015) $3101-3151$.

[34] B. Prinari, G. Biondini, D. Trubatch, Inverse scattering transform for the multi-component nonlinear Schrödinger equation with nonzero boundary conditions, Stud. Appl. Math. 126 (2011) 245-308.

[35] B. Prinari, F. Vitale, G. Biondini, Dark-bright soliton solutions with nontrivial polarization interactions for the three-component defocusing nonlinear Schrödinger equation with nonzero boundary conditions, J. Math. Phys. 56 (2015) 071505.

[36] X. Zhou, Direct and inverse scattering transforms with arbitrary spectral singularities, Comm. Pure Appl. Math., 42, 895-938 (1989)

[37] J. Villarroel, M. J. Ablowitz and B. Prinari, Solvability of the direct and inverse problems for the nonlinear Schrödinger equations, Acta Appl. Math., 87, 245-280 (2005) 
Fig. 1

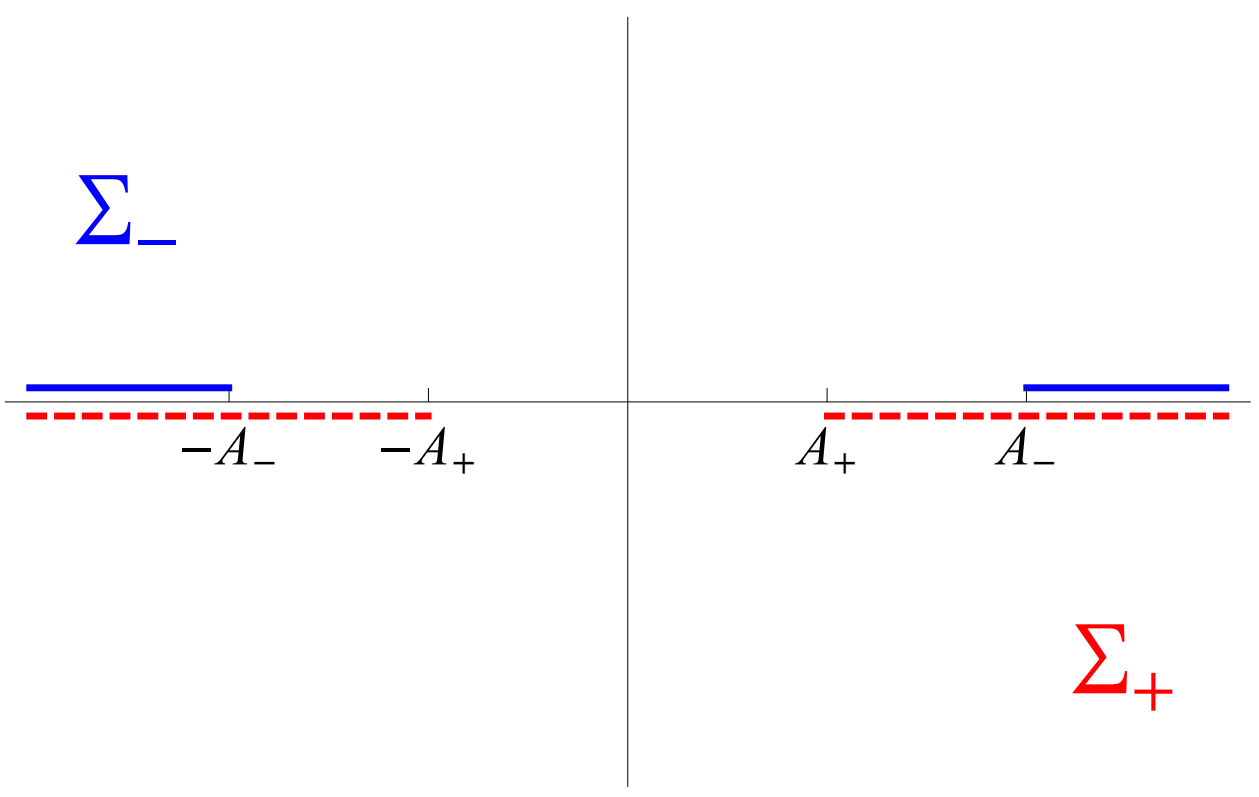

